\title{
Particle Correlations in Saturated QCD Matter
}

\author{
Rudolf Baier ${ }^{1}$, Alex Kovner ${ }^{2,3}$, Marzia Nardi ${ }^{2,45}$ and Urs Achim \\ Wiedemann ${ }^{1,2}$ \\ ${ }^{1}$ Physics Department, University of Bielefeld, D-33501 Bielefeld, Germany \\ 2 Physics Department, Theory Division, CERN, CH-1211 Geneva 23, Switzerland \\ ${ }^{3}$ Physics Department, University of Connecticut, 2152 Hillside Road, Storrs, CT \\ 06269-3046, USA \\ ${ }^{4}$ Centro Studi e Ricerche "E.Fermi" , Compendio Viminale, I-00184 Rome, Italy \\ ${ }^{5}$ INFN Sez. Torino, Via P. Giuria 1, I-10125 Torino, Italy
}

November 29, 2018

\begin{abstract}
We study quantitatively angular correlations in the two-particle spectrum produced by an energetic probe scattering off a dense hadronic target with sizeable saturation momentum. To this end, two parton inclusive cross sections for arbitrary projectiles with small color charge density are derived in the eikonal formalism. Our results are the following: For large momenta of the observed particles, the perturbative limit with characteristic back-to-back correlation is recovered. As the trigger momenta get closer to the saturation scale $Q_{s}$, the angular distribution broadens. When the momenta are significantly smaller than $Q_{s}$, the azimuthal distribution is broad but still peaked back-to-back. However, in a narrow momentum range $(0.5 \div 1.5) Q_{s}$, we observe that the azimuthal correlation splits into a double peak with maxima displaced away from $180^{\circ}$. We argue that it is the soft multiple scattering physics that is responsible for the appearance of this shift in the angle of maximal correlation. We also point out that when the physical size of the projectile is particularly small, the double peak structure persists in a significantly wider range of final state momenta.
\end{abstract}

\section{Introduction}

High- $p_{T}$ near-side and back-to-back particle correlations have become recently the focus of intensive study at the Relativistic Heavy Ion Collider (RHIC). Such correlations give access to the microscopic dynamics of two major phenomena searched for at RHIC in order to elucidate the properties of dense QCD matter [1-4]: the phenomenon of perturbative saturation [5-10] expected to determine the initial condition of hadronic collisions at sufficiently high center of mass energy, and the phenomenon of jet quenching [11-14] established to suppress the high- $p_{T}$ hadronic yields due to final state interactions. To disentangle initial and final state effects, it is clearly important to compare data on nucleus-nucleus collisions to data on proton (deuteron)-nucleus 
collisions in which final state effects are absent.

Two-particle correlations may provide further tests of the dynamics underlying final state jet quenching, since it has been argued that their angular dependence is sensitive to the density of the produced matter and its collective flow $[15,16]$. Moreover, it has been pointed out that in an ideal liquid, the energy radiated off a hard final state parton would propagate in a 'sonic boom' [17]. This would lead to an azimuthal back-to-back correlation which is peaked away from $180^{\circ}$, and may thus serve as a characteristic signature of a medium with (almost) vanishing viscosity [18]. On the other hand, it has been suggested that initial state saturation could affect dramatically the two-gluon spectrum [19] by strongly reducing the angular correlation at high transverse momentum, leading practically to a disappearance of the back-to-back correlations of jets in nuclear collisions. Although the effect is suggested to be more dramatic for large nuclear projectiles, the trend should also be seen for proton (deuteron)-nucleus collisions.

The main purpose of the present work is to study quantitatively saturation effects in the simplest process which gives rise to two-parton correlation functions. In the target rest frame, this is the emission of one gluon from a projectile quark $q A \rightarrow q g X$ with the transverse momentum of quark and gluon resolved. These results are presented in Section 2.

The other goal of this work is to set up a formalism for calculating differential multi-parton cross sections in the eikonal approximation [20]. In particular we shall show how to take properly account of the final state radiation by unitarily transforming gluonic (and in general partonic) observables with the operator which dresses the partons by the cloud of Weizsäcker-Williams gluons. We show that perturbative expansion of this cloud operator precisely generates the perturbative radiation in the final state.

The paper is organized as follows: In Section 2, we discuss the physics of two-parton correlation functions, starting from basic formulae and numerical results. The subsequent sections discuss the general framework for calculating parton correlations in the eikonal formalism and some applications. Section 3 presents the general discussion. This formalism is then applied to parton correlations for $q A \rightarrow q(\mathbf{k}) g(\mathbf{p}) X$ in Section 4 , and to $q A \rightarrow q g\left(\mathbf{p}_{1}\right) g\left(\mathbf{p}_{2}\right) X$ in Section 5 . The main results are summarized in the Conclusions.

\section{Angular correlations in $q A \rightarrow q(\mathbf{k}) g(\mathbf{p}) X$}

We consider the simplest case of a two-particle final state. The projectile consists of a single quark. It scatters on a hadronic target of sizeable saturation 
momentum and produces a gluon in the final state: $q A \rightarrow q(\mathbf{k}) g(\mathbf{p}) X$. For a related discussion with strong emphasis on breaking of $k_{T}$-factorization, see Refs. [21-24].

\section{A. Basic formulae and definitions}

The starting point of our study is the following expression for the doubly inclusive spectrum $q A \rightarrow q(\mathbf{k}) g(\mathbf{p}) X$ (for derivation see Sections 3 and 4 )

$$
\begin{aligned}
\frac{d N}{d y d \mathbf{k} d \mathbf{p}}=\frac{\alpha_{s} C_{F}}{\pi^{2}} & \frac{1}{(2 \pi)^{4}} \int d \mathbf{z} d \overline{\mathbf{z}} d \mathbf{x} e^{-i \mathbf{k} \cdot \mathbf{x}-i \mathbf{p} \cdot(\mathbf{z}-\overline{\mathbf{z}})} \frac{(\mathbf{x}-\mathbf{z}) \cdot \overline{\mathbf{z}}}{(\mathbf{x}-\mathbf{z})^{2} \overline{\mathbf{z}}^{2}} \\
\times & {[Q(\mathbf{z}, \mathbf{x}, \mathbf{0}, \overline{\mathbf{z}}) S(\overline{\mathbf{z}}, \mathbf{z})+S(\mathbf{x}, \mathbf{0})} \\
& -S(\mathbf{x}, \overline{\mathbf{z}}) S(\overline{\mathbf{z}}, \mathbf{0})-S(\mathbf{x}, \mathbf{z}) S(\mathbf{z}, \mathbf{0})]
\end{aligned}
$$

The entire information about the target nucleus is contained in two target averages of products of Wilson lines,

$$
\begin{aligned}
S(\overline{\mathbf{u}}, \mathbf{u}) & =\left\langle\frac{1}{N} \operatorname{Tr}\left[W^{F^{\dagger}}(\overline{\mathbf{u}}) W^{F}(\mathbf{u})\right]\right\rangle_{T} \\
Q(\overline{\mathbf{u}}, \mathbf{u}, \mathbf{z}, \overline{\mathbf{z}}) & =\left\langle\frac{1}{N} \operatorname{Tr}\left[W^{F^{\dagger}}(\overline{\mathbf{u}}) W^{F}(\mathbf{u}) W^{F^{\dagger}}(\mathbf{z}) W^{F}(\overline{\mathbf{z}})\right]\right\rangle_{T} .
\end{aligned}
$$

To model these target averages, we use the expressions $[5,21]$

$$
\begin{aligned}
& S(\overline{\mathbf{u}}, \mathbf{u})= \exp [-v(\overline{\mathbf{u}}-\mathbf{u})] \\
& Q(\overline{\mathbf{y}}, \mathbf{x}, \overline{\mathbf{x}}, \mathbf{y})=\frac{v(\mathbf{x}-\overline{\mathbf{x}})+v(\mathbf{y}-\overline{\mathbf{y}})-v(\mathbf{x}-\mathbf{y})-v(\overline{\mathbf{x}}-\overline{\mathbf{y}})}{v(\mathbf{x}-\overline{\mathbf{x}})+v(\mathbf{y}-\overline{\mathbf{y}})-v(\mathbf{x}-\overline{\mathbf{y}})-v(\mathbf{y}-\overline{\mathbf{x}})} \\
& \quad \quad \quad \exp [-v(\mathbf{x}-\overline{\mathbf{x}})-v(\mathbf{y}-\overline{\mathbf{y}})] \\
& \quad-\frac{v(\mathbf{x}-\overline{\mathbf{y}})+v(\mathbf{y}-\overline{\mathbf{x}})-v(\mathbf{x}-\mathbf{y})-v(\overline{\mathbf{x}}-\overline{\mathbf{y}})}{v(\mathbf{x}-\overline{\mathbf{x}})+v(\mathbf{y}-\overline{\mathbf{y}})-v(\mathbf{x}-\overline{\mathbf{y}})-v(\mathbf{y}-\overline{\mathbf{x}})} \\
& \quad \times \exp [-v(\mathbf{x}-\overline{\mathbf{y}})-v(\mathbf{y}-\overline{\mathbf{x}})] \\
& v(\mathbf{x})=\mathbf{x}^{2} \frac{\tilde{Q}_{s}^{2}(\mathbf{x})}{8} \equiv \mathbf{x}^{2} \frac{Q_{s, 0}^{2}}{8} \log \left[\frac{1}{\mathbf{x}^{2} \Lambda^{2}}+a\right]
\end{aligned}
$$

The function $v(\mathbf{x})$ has the meaning of the target gluon field correlation function and is directly proportional to the gluon density in the target, hence the logarithmic dependence on the transverse separation.

For numerical evaluation, we shall take $\Lambda \equiv \Lambda_{\mathrm{QCD}}=0.2 \mathrm{GeV}$. The small regulator $a=1 /\left(x_{c}^{2} \Lambda^{2}\right), x_{c}=3 \mathrm{GeV}^{-1}$ is chosen such that the logarithm in (2.6) does not turn negative and that the sensitivity on the infrared cut-off 
is negligible for sufficiently large momentum [25]. The saturation scale $Q_{s}$ is defined implicitly as

$$
Q_{s}^{2} \equiv \tilde{Q}_{s}^{2}\left(\mathbf{x}^{2}=1 / Q_{s}^{2}\right)
$$

With this definition, $Q_{s}^{2}=2 \mathrm{GeV}^{2}$ corresponds to $Q_{s, 0}^{2} \simeq 0.5 \mathrm{GeV}^{2}$ in Eq. (2.6).

In the model (2.4)-(2.6) the properties of the single inclusive gluon radiation spectrum $q A \rightarrow q g(\mathbf{p}) X$ and its small- $x$ evolution have been studied extensively in recent years and are well understood [25-29]. This spectrum is obtained from the two-particle correlation function (2.1), integrating over the quark momentum $\mathbf{k}$,

$$
\begin{aligned}
\frac{d N}{d y d \mathbf{p}}=\frac{\alpha_{s} C_{F}}{\pi^{2}} & \frac{1}{(2 \pi)^{2}} \int d \mathbf{z} d \overline{\mathbf{z}} e^{-i \mathbf{p} \cdot(\mathbf{z}-\overline{\mathbf{z}})} \frac{\mathbf{z} \cdot \overline{\mathbf{z}}}{\mathbf{z}^{2} \overline{\mathbf{z}}^{2}} \\
\times & {\left[S^{2}(\overline{\mathbf{z}}, \mathbf{z})+1-S^{2}(\overline{\mathbf{z}})-S^{2}(\mathbf{z})\right] . }
\end{aligned}
$$

In the approximation (2.4)-(2.6), one finds

$$
\begin{aligned}
\frac{d N}{d y d \mathbf{p}}= & \frac{1}{\pi} \int d \mathbf{z} d \overline{\mathbf{z}} \frac{1}{(2 \pi)^{2}} \frac{\alpha_{s} C_{F}}{\pi} \frac{\mathbf{z} \cdot \overline{\mathbf{z}}}{\mathbf{z}^{2} \overline{\mathbf{z}}^{2}} e^{-i \mathbf{p} \cdot(\mathbf{z}-\overline{\mathbf{z}})} \\
& \times\left[1+e^{-2 v(\mathbf{z}-\overline{\mathbf{z}})}-e^{-2 v(\mathbf{z})}-e^{-2 v(\overline{\mathbf{z}})}\right]
\end{aligned}
$$

which coincides with the quasiclassical expression of [30].

In the limit of small transverse momenta, the relevant values of the transverse coordinates $\mathbf{z}, \overline{\mathbf{z}}$ are large. In this case, the logarithm in (2.6) becomes unimportant and the Gaussian approximation $Q_{s}^{2}(\mathbf{x})=\bar{Q}_{s}^{2}$ is justified. Formally, one finds in this approximation

$$
\left.\frac{d N}{d y d \mathbf{p}}\right|_{\text {Gaussian }}=\frac{\alpha_{s} C_{F}}{\pi^{3} \bar{Q}_{s}^{2}} \int d \mathbf{q} e^{-\mathbf{q}^{2} / \bar{Q}_{s}^{2}} \frac{\mathbf{q}^{2}}{\mathbf{p}^{2}(\mathbf{p}-\mathbf{q})^{2}}
$$

Here, $\frac{\mathbf{q}^{2}}{\mathbf{p}^{2}(\mathbf{p}-\mathbf{q})^{2}}$ is the typical momentum dependence of gluons radiated off a high-energy quark, which received a momentum transfer q. For gluons of small transverse momentum $\mathbf{p}$, the momentum transfer $\mathbf{q}$ from the target is accumulated according to transverse Brownian motion $\left\langle\mathbf{q}^{2}\right\rangle \propto \bar{Q}_{s}^{2} \propto A^{1 / 3}$. For a hard gluon $|\mathbf{p}| \gg Q_{s}$, however, Eq. (2.9) shows the typical power law $\propto \frac{1}{\mathbf{p}^{4}} \ln \frac{\mathbf{p}^{2}}{\Lambda^{2}}$ characteristic for a single hard scattering off the power-law tail of a hard scattering center, see (2.15) below. In this way, the ansatz (2.6) for the saturation momentum characterizes a medium with a physically sensible 
interpolation between single hard and multiple soft scattering as a function of transverse momentum transfer. We note that this is the QCD analogue of QED Molière scattering theory [31], which achieves the analogous interpolation between soft multiple and single hard target momentum transfers for electromagnetic scattering processes.

\section{B. Perturbative baseline}

As a baseline for comparison of our numerical results, we collect here the perturbative expressions for the single and double inclusive cross sections. These do not include the effects of multiple rescattering which are due to the existence of a large saturation scale in the target. The perturbative baseline is obtained by identifying the single hard interaction term in the target averages (2.4), (2.5). This corresponds to an expansion to first order in $v(\mathbf{x})$ :

$$
\begin{aligned}
S(\overline{\mathbf{u}}, \mathbf{u})=1- & v(\overline{\mathbf{u}}-\mathbf{u})+O\left(v^{2}\right) \\
Q(\overline{\mathbf{u}}, \mathbf{u}, \overline{\mathbf{w}}, \mathbf{w})=1-[ & v(\overline{\mathbf{u}}-\mathbf{u})+v(\overline{\mathbf{w}}-\mathbf{w})+v(\overline{\mathbf{u}}-\mathbf{w})+v(\overline{\mathbf{w}}-\mathbf{u}) \\
& -v(\mathbf{u}-\mathbf{w})-v(\overline{\mathbf{u}}-\overline{\mathbf{w}})]+O\left(v^{2}\right) .
\end{aligned}
$$

Inserting this into Eq.(2.1), we find

$$
\frac{d N}{d y d \mathbf{k} d \mathbf{p}}=\frac{\alpha_{s} C_{F}}{\pi^{2}} \frac{Q_{s, 0}^{2}}{\pi} \frac{1}{\mathbf{p}^{2}(\mathbf{k}+\mathbf{p})^{2} \mathbf{k}^{2}}
$$

To obtain (2.13), one has to keep the logarithmic dependence in the scale entering $v(\mathbf{x})$ in (2.6). Neglecting this dependence of $Q_{s}$ on $\mathbf{x}$ amounts to neglecting the possibly large (albeit more rare) momentum transfer due to the interaction with the high momentum tail of the target fields. In this case, $Q_{s}^{2}(\mathbf{x})=\bar{Q}_{s}^{2}$, one finds instead

$$
\frac{d N_{\mathrm{Gauss}}}{d y d \mathbf{k} d \mathbf{p}}=\frac{\alpha_{s} C_{F}}{\pi^{2}} \frac{\bar{Q}_{s}^{2}}{\mathbf{p}^{4}} \delta^{(2)}(\mathbf{p}+\mathbf{k}),
$$

where the quark and gluon momenta are exactly balanced. This shows that as long as the interaction with the target can not impart a significant kick to a propagating parton, large momenta in the final state can only appear due to the large relative momentum between the quark and the gluon in the initial wave function of the projectile.

Integrating Eq.(2.13) over the quark mometum $\mathbf{k}$, we obtain the perturbative expression for the single gluon inclusive cross section 


$$
\left.\frac{d N}{d y d \mathbf{p}}\right|_{|\mathbf{p}| \gg Q_{s}}=\frac{2 \alpha_{s} C_{F} Q_{s, 0}^{2}}{\pi^{2}} \frac{1}{\mathbf{p}^{4}}\left(\ln \left[\frac{\mathbf{p}^{2}}{4 \Lambda^{2}}\right]+2 \gamma_{E}-1\right)
$$

To determine the distribution of the total recoil momentum, we integrate (2.1) over the relative momentum of the quark-gluon pair in the final state. Defining

$$
\mathbf{K}=\mathbf{k}+\mathbf{p}, \quad \mathbf{q}=\mathbf{k}-\mathbf{p}
$$

we find to leading logarithmic accuracy

$$
\frac{d N}{d y d \mathbf{K}}=2 \frac{\alpha_{s} C_{F}}{\pi^{2}} \frac{Q_{s, 0}^{2}}{\left(\mathbf{K}^{2}\right)^{2}} \ln \left[\frac{\mathbf{K}^{2}}{\Lambda^{2}}\right]
$$

This expression exhibits the typical perturbative power-law dependence for the total recoil momentum $K$ above the infrared regulator scale $\Lambda$. This is consistent with the expectation, that for large recoil $\mathbf{K}$, the target behaves like a single hard perturbative scattering center.

We also give here the perturbative expression for the two gluon inclusive cross section $q A \rightarrow q g\left(\mathbf{p}_{1}\right) g\left(\mathbf{p}_{2}\right) X$, with gluons produced at rapidities $\eta$ and $\xi$. The full expression, including the effects of the saturation is given in Eq.(5.14) below. Its perturbative limit is

$$
\frac{d N}{d \eta d \mathbf{p}_{1} d \xi d \mathbf{p}_{2}}=\frac{\alpha_{s}^{2}}{\pi^{6}} Q_{s, 0}^{2} \frac{1}{\mathbf{p}_{1}^{2} \mathbf{p}_{2}^{2}\left(\mathbf{p}_{1}+\mathbf{p}_{2}\right)^{2}} \ln \frac{\left(\mathbf{p}_{1}+\mathbf{p}_{2}\right)^{2}}{\Lambda^{2}}
$$

\section{Distribution of the recoil momentum}

The total transverse momentum $\mathbf{K}$ of the quark-gluon pair in the final state traces the distribution of recoil momentum transferred from the target. We calculate it by integrating (2.1) over the relative transverse momentum q. After performing some angular integrations, one finds

$$
\begin{aligned}
\frac{d N}{d y K d K}=\frac{\alpha_{s} C_{F}}{\pi^{2}} & \int_{0}^{\infty} d z \int_{0}^{1 / \Lambda_{\mathrm{cut}}} d \bar{z} \int_{0}^{2 \pi} d \phi \frac{z}{\bar{z}} J_{0}(K z) \\
\times & {[Q(\mathbf{z}+\overline{\mathbf{z}}, \mathbf{z}, \mathbf{0}, \overline{\mathbf{z}}) S(\mathbf{z})+S(\mathbf{z})} \\
& -S(\mathbf{z}-\overline{\mathbf{z}}) S(\overline{\mathbf{z}})-S(\overline{\mathbf{z}}) S(\mathbf{z}+\overline{\mathbf{z}})]
\end{aligned}
$$

Here, we have introduced the infrared cut-off $\Lambda_{\text {cut }}$ to regulate the logarithmically infrared divergent $\bar{z}$-integral. For the discussion in this subsection, we 
choose $\Lambda_{\text {cut }}=\Lambda$, the same infrared cut-off, which regulates the perturbative expression (2.17). However, as discussed below, $\Lambda_{\text {cut }}$ has a physical interpretation as regulator of the transverse size of the incoming projectile. We will discuss the implications of restricing the size of the projectile in more detail later.

We have evaluated expression (2.19) numerically. In Fig. 1 we plot the ratio of the distribution (2.19) to the perturbative expression (2.17). For momenta $K \gg Q_{s}$ well above the saturation scale, the recoil distribution (2.19) approaches the perturbative power law $\propto \frac{1}{K^{4}} \ln \frac{K^{2}}{\Lambda^{2}}$ and is indistinguishable from the perturbative expression. For low momenta $K<Q_{s}$, the saturated distribution is suppressed relative to the perturbative one. This is the manifestation of the fact that a parton propagating through the saturated target is very unlikely to get a kick of momentum less than of order of $Q_{s}$, whereas in the interaction with the perturbative target on the contrary low momentum transfer processes are very important. Finally, Fig. 1 exhibits a fairly wide maximum around $K=(2 \div 3) Q_{s}$, which is due to the transfer of momentum of order $Q_{s}$ to one of the propagating partons ( $q$ or $g$ ). The general features of Fig. 1 are very similar to those of the nuclear modification factor calculated within the same model in $[25,28,29]$.

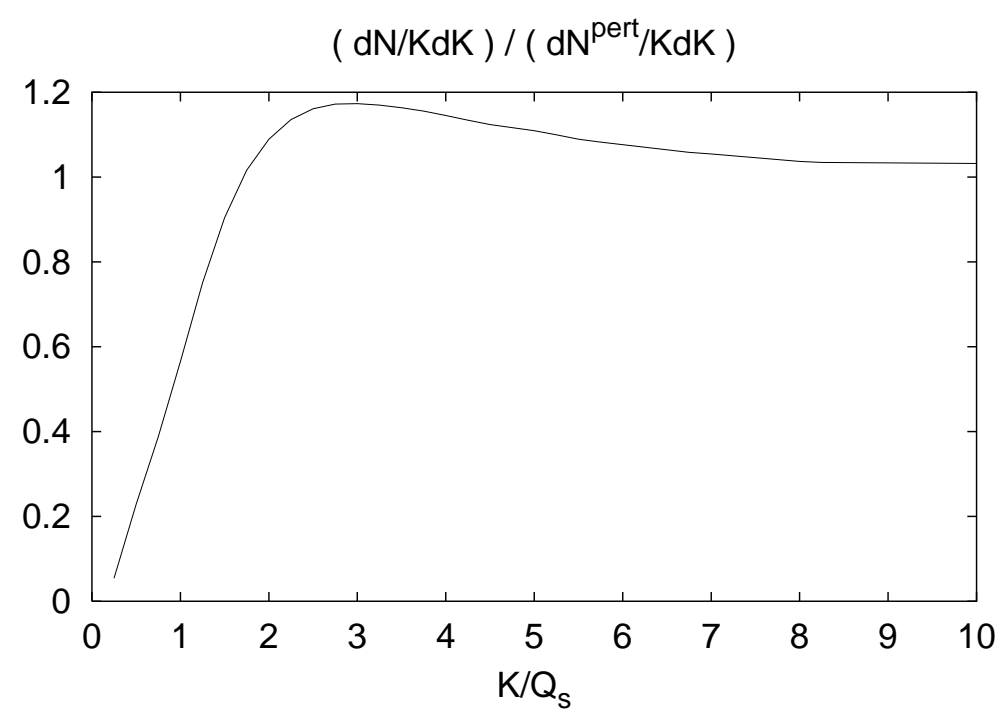

Fig. 1. The full two-parton (quark-gluon) correlator (2.19) normalized to its perturbative limit (2.17) as a function of the total pair momentum $K=|\mathbf{k}+\mathbf{p}|$.

\section{Angular Correlations - large trigger momentum}

We next study the angular dependence of the two-parton correlation function (2.1). Shifting $\mathbf{z} \rightarrow \mathbf{z}+\mathbf{x}$ and introducing radial coordinates, we find 


$$
\begin{aligned}
& \frac{d N}{d y k d k p d p d \Delta \phi_{k p}}= \frac{\alpha_{s} C_{F}}{\pi^{2}} \frac{1}{(2 \pi)^{2}} \int x d x \int^{\frac{1}{\Lambda_{\mathrm{cut}}}} d z \int^{\frac{1}{\Lambda_{\mathrm{cut}}}} d \bar{z} \\
& \times \int d \phi_{z} d \phi_{\bar{z}} \cos \left(\phi_{z}-\phi_{\bar{z}}\right) J_{0}\left(\sqrt{A^{2}+B^{2}}\right) \\
& \times[Q(\mathbf{z}+\mathbf{x}, \mathbf{x}, \mathbf{0}, \overline{\mathbf{z}}) S(\overline{\mathbf{z}}, \mathbf{z}+\mathbf{x})+S(\mathbf{x}, \mathbf{0}) \\
&\quad-S(\mathbf{x}, \overline{\mathbf{z}}) S(\overline{\mathbf{z}}, \mathbf{0})-S(\mathbf{0}, \mathbf{z}) S(\mathbf{z}+\mathbf{x}, \mathbf{0})]
\end{aligned}
$$

Here, $\mathbf{z} \cdot \overline{\mathbf{z}}=z \bar{z} \cos \left(\phi_{z}-\phi_{\bar{z}}\right), \mathbf{x} \cdot \mathbf{z}=x z \cos \left(\phi_{z}\right), \overline{\mathbf{z}} \cdot \mathbf{x}=\bar{z} x \cos \left(\phi_{\bar{z}}\right)$ and the notational shorthands

$$
\begin{aligned}
& A=k x \cos \left(\Delta \phi_{k p}\right)+p x+p z \cos \left(\phi_{z}\right)-p \bar{z} \cos \left(\phi_{\bar{z}}\right), \\
& B=k x \sin \left(\Delta \phi_{k p}\right)-p z \sin \left(\phi_{z}\right)+p \bar{z} \sin \left(\phi_{\bar{z}}\right) .
\end{aligned}
$$

The $z$ and $\bar{z}$ integrals in equation (2.20) are logarithmically IR divergent. Regulating these integrals by cutting off both integrations at distances greater than $1 / \Lambda_{\text {cut }}$, we can numerically determine the correlation of the two partons as a function of their relative azimuthal angle $\Delta \phi_{k p}$ and their transverse momentum $k$ and $p$. Technically, it is sufficient to regulate either one of the integrations $z$ or $\bar{z}$, to arrive at a finite result. Physically, the cut-off $1 / \Lambda_{\text {cut }}$ limits the transverse size of the incoming wave function to $z<1 / \Lambda_{\text {cut }}$ in the amplitude and to $\bar{z}<1 / \Lambda_{\text {cut }}$ in the complex conjugate amplitude. Thus, we choose to implement the cut-off in a symmetric way.

Fig. 2(a) shows the correlation function as a function of the azimuthal angle for a fixed and relatively large value of the quark momentum $k=4 Q_{s}$ and values of the gluon momentum $p$ between $Q_{s}$ and $4 Q_{s}$. We observe that for larger values of $p$ the distribution exhibits a strong back-to-back correlation. As $p$ decreases, the distribution becomes wider, and finally at $p=Q_{s}$ it is practically flat.

The main features of Fig. 2(a) can be understood in the following simple picture. The incoming wave function of the projectile in the present approximation contains only one quark and up to one gluon (see Sections 3-5). The total transverse momentum of the incoming quark-gluon system is zero, and thus they are exactly correlated back-to-back. While travelling through the target, each parton gets a transverse kick which in most cases is close to $Q_{s}$. However, albeit with small probability, the impinging partons can scatter off the perturbative high momentum tail of the target field (Molière scattering). The final state configurations with large transverse momentum of both, quark and gluon, come mainly from two sources: either from the wave function components of the projectile where $q$ and $g$ have both large momentum and experience soft momentum transfer, or from a low momentum component of the incoming wave function where the quark experiences hard scattering off the Molière tail and subsequently radiates a gluon in the final state. [In 
the present calculation only the scattered quark can radiate. The radiation off the gluon is higher order correction in $\alpha_{s}$, since the weight of the gluon component in the initial state itself is of order $\alpha_{s}$.] These events are therefore mostly back-to-back correlated, mirroring correlations in the initial projectile wave function and the back-to-back nature of the final state radiation.
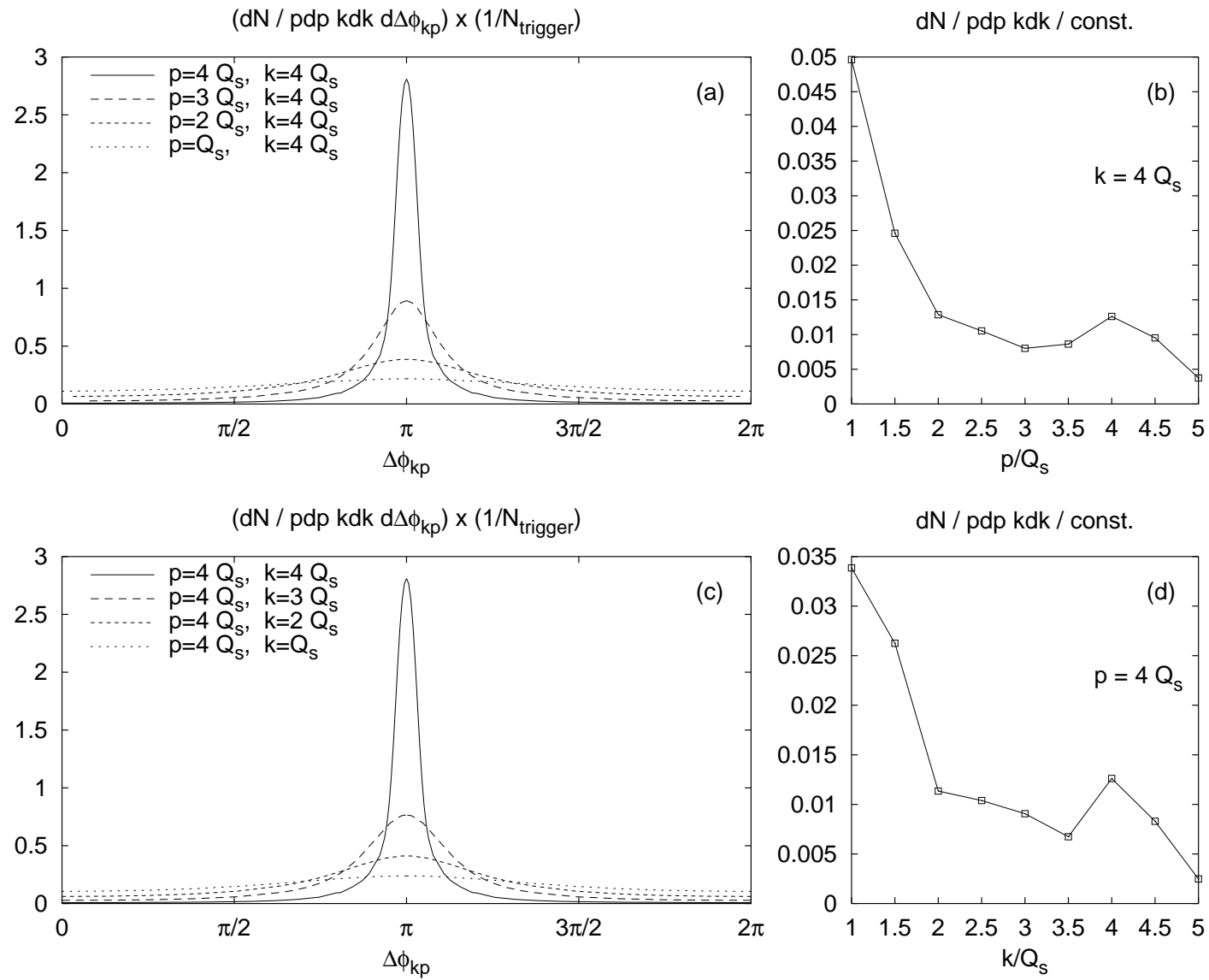

Fig. 2. (a),(c): The azimuthal dependence of the quark-gluon correlator (2.20) for different values of quark $(k)$ and gluon $(p)$ transverse momentum. The area under the curves is normalized to one, as required for differential rates per trigger particle. (b),(d): The value of the $\Delta \Phi_{k p}$-integrated double differential two-parton yield $(2.20)$, const. $=\frac{\alpha_{s} C_{F}}{\pi^{2}} \frac{1}{(2 \pi)^{2}}$.

On the other hand, final states with a large transverse momentum of the quark but a gluon with $p=Q_{s}$ can only arise due to Molière scattering of the quark, since there are no asymmetric configurations in the initial projectile wave function. The direction of the hard kick the quark experiences is random and uncorrelated with the direction of momentum of the gluon, and thus the angular distribution in this regime is almost completely flat, see Fig. 2(a). For the intermediate values of $p$, the distribution interpolates between these two extremes. 
The angular integrated intensity (2.20) is shown in Fig. 2(b) as a function of $p$ for a trigger quark momentum $k=4 Q_{s}$. It exhibits a characteristic maximum at $p=0$ and another less sharp maximum at $p=k$. The maximum at $p=0$ originates from the momentum distribution of the incoming projectile wave function, which is peaked at small values of momentum as $1 / p^{2}$. The behavior near this peak is unaffected by the presence of $Q_{s}$, but it is affected by the infrared cut-off $\Lambda_{\text {cut }}$, which eliminates the low relative momentum configurations from the initial wave function. The maximum at $p=k$ can be traced to the pertubative expression (2.13) which (after integration over $\Delta \phi_{k p}$ ) diverges linearly at $p=k$. This divergence originates from the back-to-back correlations between the quark and the gluon in the projectile wave function and the back-to-back final state radiation. For the saturated target however, the final state momentum of each parton is smeared around its initial state value within an interval of the width $\pm Q_{s}$. Thus the linearly divergent peak of (2.13) turns into a finite peak of width $Q_{s}$ in Fig. 2(b).

Figs. 2(c,d) present analogous plots, where the momentum of the outgoing gluon is fixed at $p=4 Q_{s}$ and the quark momentum is varied. Although some fine details are different, the overall picture is qualitatively very similar to Figs. 2(a,b).

\section{E. Angular Correlations - trigger momenta close to $Q_{s}$}

We consider now the regime where the trigger momenta are of the order of $Q_{s}$. We concentrate on the situation when the associated momenta are equal, $p=k$. In Fig. 3, we show angular correlations when the trigger momenta are varied between $0.625 Q_{s}$ and $1.5 Q_{s}$. These plots show a structure distinct from the expected back-to-back correlations. The angular correlation is not peaked at $\phi=\pi$, but instead at $\phi=\pi-\delta$, where $\delta$ is clearly greater for smaller values of $p$. The dip at $\phi=\pi$ disappears for trigger momenta lower than about $0.6 Q_{s}$ and higher than about $1.4 Q_{s}$.

The dip at $\pi$ arises due to a coherent scattering effect, namely due to soft multiple scattering of the initial quark-gluon components of small transverse size. If the size of the pair is smaller than the transverse correlation length of the target fields, then we expect that the pair scatters as one single object as it sees the same target fields. It then picks up a typical soft momentum which is shared equally between the quark and the gluon. [In the model defined by (2.6), it seems reasonable to use $Q_{s, 0}$ as the typical soft scale, since the logarithmic correction which shifts $Q_{s, 0}$ to $Q_{s}$ is due to harder target gluons. Also, the correlation length of the target may be estimated to be of order $\left.1 / Q_{s, 0} \cdot\right]$ In the initial state the quark and the gluon have momenta equal in magnitude and opposite in direction. In our trigger momenta kinematics, 
where the magnitudes of the trigger momenta are also equal, the momentum transfer from the target must be in the direction perpendicular to the initial momenta. We can thus estimate the maximal correlation angle when $p>Q_{s, 0}$ by the following argument. We start with the initial momenta of the quark and the gluon $\mathbf{k}_{i n},-\mathbf{k}_{i n}$. In the final state $\mathbf{k}=\mathbf{k}_{i n}+\delta \mathbf{k}$ and $\mathbf{p}=-\mathbf{k}_{\text {in }}+\delta \mathbf{k}$ with $|\delta \mathbf{k}|=Q_{s, 0} / 2$ and $\mathbf{k}_{i n} \cdot \delta \mathbf{k}=0$. For $k \gg Q_{s, 0}$ we find the angle between the momenta in the final state

$$
\phi=\pi-\frac{Q_{s, 0}}{\sqrt{2} k} .
$$

The magnitude of the effect seen in Fig. 3 is consistent with this rough estimate.

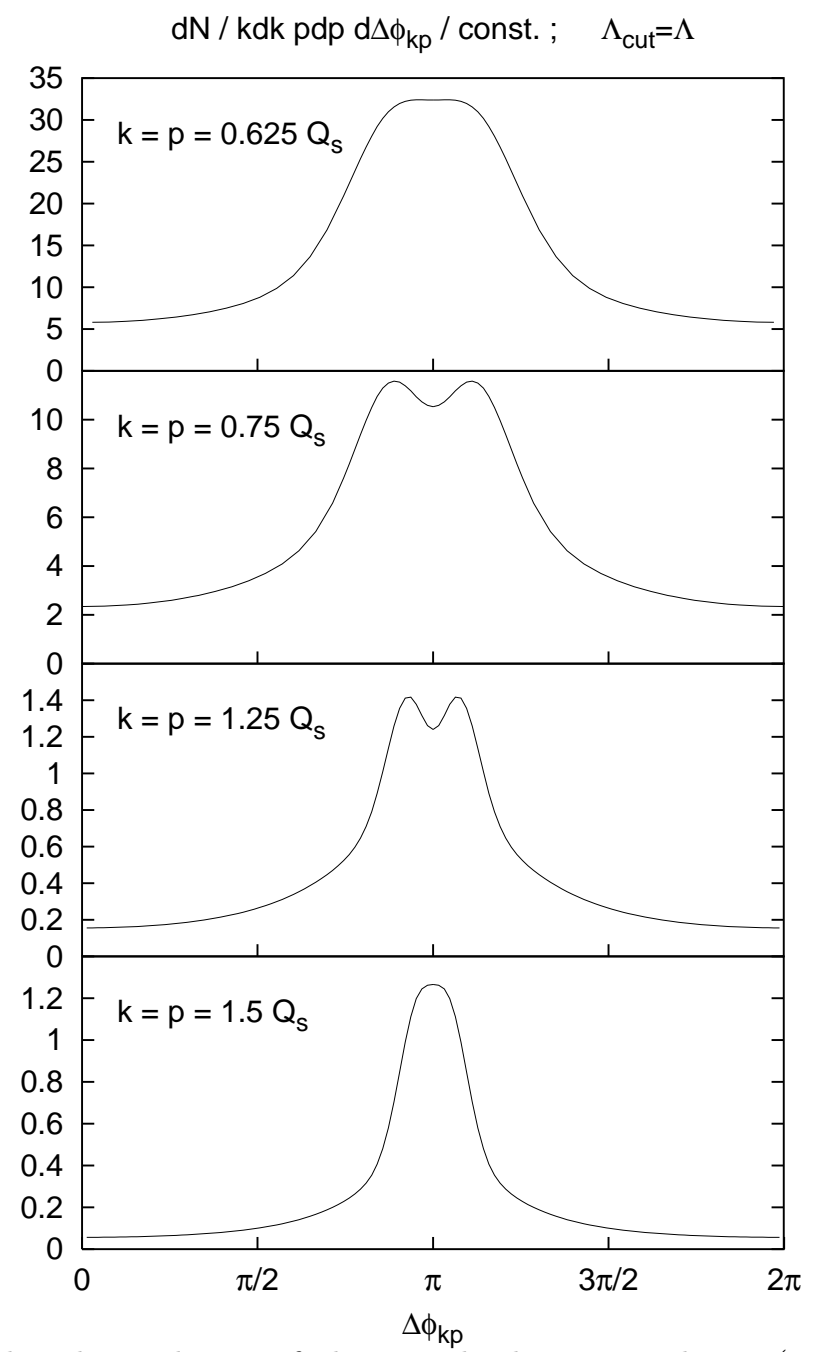

Fig. 3. Angular dependence of the quark-gluon correlator (2.20) for different values of the trigger momentum close to $Q_{s}$, const. $=\frac{\alpha_{s} C_{F}}{\pi^{2}} \frac{1}{(2 \pi)^{2}}$. 
So far, we have discussed how coherent scattering of incoming parton pairs can lead to a shift of the backward peak away of zero. To understand why the dip in the two-parton correlator appears only for a narrow range of trigger momenta, we consider two other scattering mechanisms: i) the perturbative hard Molière scattering off the large momentum tails of the target, which leads to a back-to-back distribution, and ii) the incoherent soft scattering for those components of the wave function, whose transverse size is greater than the correlation length $\sim 1 / Q_{s, 0}$ of the target fields. Indeed, when the trigger momenta are much higher than $Q_{s}$, the phase space in the initial state from which one can get to these final momenta by the coherent scattering mechanism is very small. Thus the perturbative final state radiation dominates, which is exactly back-to-back. The phase space for coherent scattering of incoming parton pairs also disappears if $k_{\text {trigger }}$ is significantly smaller than $Q_{s}$, since the coherent scattering mechanism requires enough particles in the initial state with momenta $k_{\text {initial }}<k_{\text {trigger }}-\beta Q_{s}$ with $\beta$ - a number of order one. Thus, it seems natural that the coherent scattering mechanism is dominant only for trigger momenta around $Q_{s}$.

To further test the mechanism suggested to underly the dip in the two-parton correlation functions, one can exploit the dependence of the two-parton correlation function (2.20) on the infrared cut-off $\Lambda_{\text {cut }}$. In the derivation of this expression in Section 4, this cut-off dependence can be seen to restrict the cross section of the final state radiation. Thus, if one artificially raises the infrared cut-off so that it is close to the trigger momentum, one expects that most of the contribution of the back-to-back correlated, final state radiation is eliminated. One expects a dip at $\phi=\pi$ for a much larger trigger momentum, with maxima at a position given by the simple estimate (2.23). We have performed various such consistency checks to further substantiate the explanation given above and find that the results conform with this simple picture.

The physical interpretation to the cut-off dependence of (2.20) suggests that one can use it to extract some additional information from our calculation. As mentioned above, and as can be seen from the derivation in Section 4, the cut-off on the $z$ and $\bar{z}$ integrals restricts the size of the projectile wave function to $1 / \Lambda_{\text {cut }}$. Thus, varying the cut-off, we can probe the physics of small size projectiles. For example, since the scale of 0.3 Fermi is the natural size of the constituent quark, one may ask what is the effect of restricting the gluon in the wave function to be emitted no further than 0.3 Fermi from the valence quark. [Alternatively, one may think of this excercise as a "poor man's" way to mimick the doubly inclusive spectrum of a scattering of a dipole of this size.] In any case, raising the cut-off to this value should give a reasonable indication of the upper limit on the effect that one might expect. The numerical results with the cut-off $\Lambda_{\text {cut }}=Q_{s, 0}=3.57 \Lambda$ are shown in Fig. 4 . In accordance with our expectations, the effect is more pronounced. The dip at $\phi=\pi$ now persists up to higher values of the trigger momentum, about $2.5 Q_{s}$. It is also much 
deeper than for the lower IR cut-off.

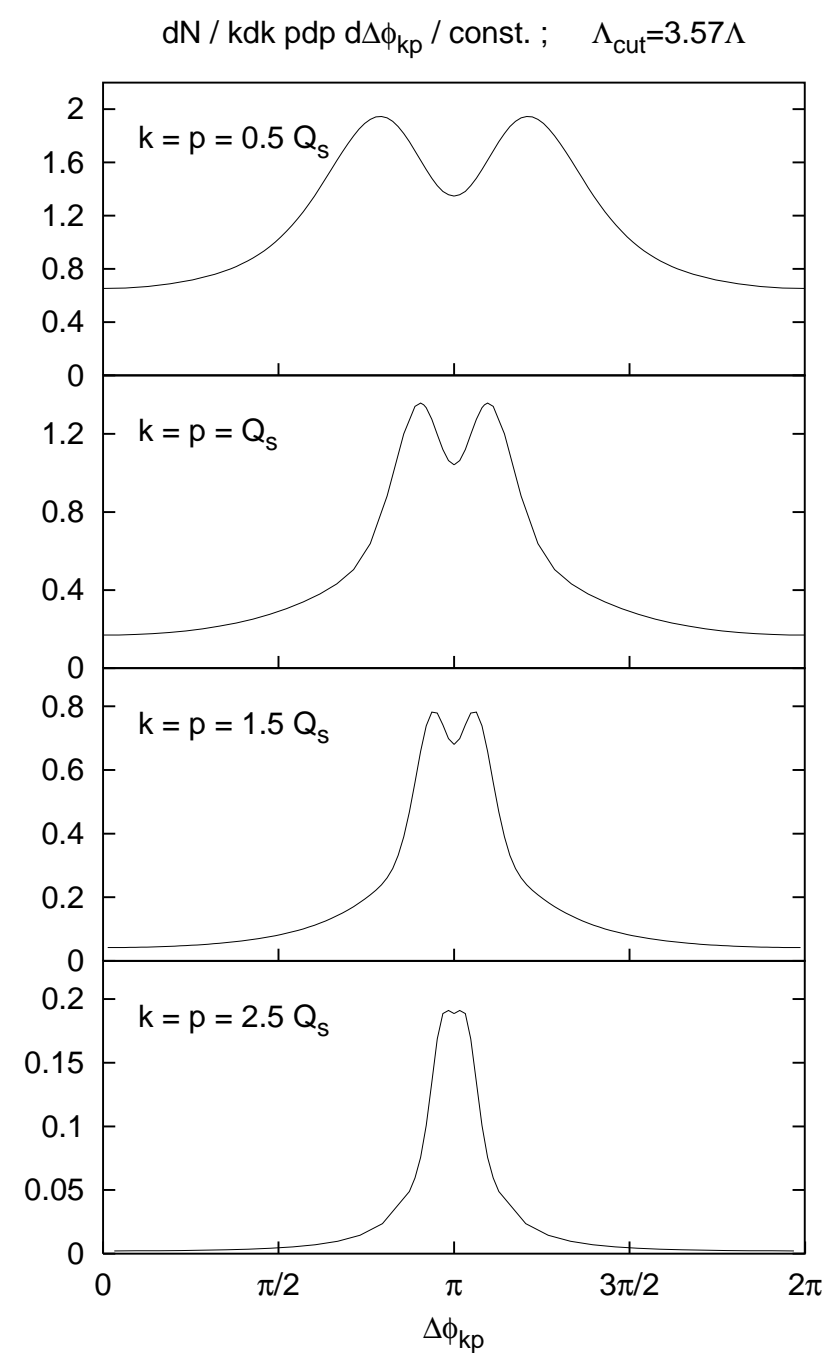

Fig. 4. Angular dependence of the quark-gluon correlator (2.20) for different values of the trigger momentum close to $Q_{s}$, const. $=\frac{\alpha_{s} C_{F}}{\pi^{2}} \frac{1}{(2 \pi)^{2}}$. Here, the transverse size of the incoming projectile wavefunction is restricted to a small value $1 / \Lambda_{\text {cut }}$.

This concludes our discussion of the numerical results. We now turn to the derivation of the basic formulae used in this section and a more general discussion of differential cross sections in the eikonal formalism.

\section{Differential cross sections in the eikonal approximation}

Below we follow the basic formalism of [20]. Consider an energetic hadronic projectile impinging on a large nuclear target. The projectile is characterized 
by a wave function, in which the relevant degrees of freedom are the transverse positions and color states of the partons,

$$
\left|\Psi_{i n}\right\rangle=\sum_{\left\{\alpha_{i}, \mathbf{x}_{i}\right\}} \psi\left(\left\{\alpha_{i}, \mathbf{x}_{i}\right\}\right)\left|\left\{\alpha_{i}, \mathbf{x}_{i}\right\}\right\rangle
$$

The color index $\alpha_{i}$ can belong to the fundamental, antifundamental or adjoint representation of the color $S U\left(N_{c}\right)$ group, corresponding to quark, antiquark or gluon in the wavefunction. In what follows we will consider wave functions with a small number of partons.

At high energy, the propagation time through the target is short, and thus partons propagate independently of each other. For the same reason the transverse positions of the partons do not change during the propagation. The only effect of the propagation is that the wave function of each parton acquires an eikonal phase due to the interaction with the gluon field of the target. Thus the projectile emerges form the interaction region with the wave function

$$
\left|\Psi_{\text {out }}\right\rangle=\mathcal{S}\left|\Psi_{\text {in }}\right\rangle=\sum_{\left\{\alpha_{i}, \mathbf{x}_{i}\right\}} \psi\left(\left\{\alpha_{i}, \mathbf{x}_{i}\right\}\right) \prod_{i} W\left(\mathbf{x}_{i}\right)_{\alpha_{i} \beta_{i}}\left|\left\{\beta_{i}, \mathbf{x}_{i}\right\}\right\rangle .
$$

Here $\mathcal{S}$ is the $S$-matrix, and the $W$ 's are Wilson lines

$$
W\left(\mathbf{x}_{i}\right)=\mathcal{P} \exp \left\{i \int d z^{-} T^{a} A_{a}^{+}\left(\mathbf{x}_{i}, z^{-}\right)\right\}
$$

with $A^{+}$- the gauge field in the target and $T^{a}$ - the generator of $S U\left(N_{c}\right)$ in a representation corresponding to a given parton. The relative phases between the components of the wave function change, and the state that emerges after the target is no longer an eigenstate of the strong interaction Hamiltonian (as the incoming state is assumed to be) but rather a superposition of such eigenstates. It is convenient to rewrite the outgoing wave function in terms of the second quantized operator corresponding to the gauge rotation Eq.(3.3)

$$
\left|\Psi_{\text {out }}\right\rangle=\hat{W}\left|\Psi_{\text {in }}\right\rangle
$$

with

$$
\hat{W}=\exp \left[i \int \lambda^{a}(\mathbf{x}) \rho^{a}(\mathbf{x})\right]
$$

Here $\rho^{a}(\mathbf{x})$ is the color charge density operator, integrated over the rapidities of the projectile, and the parameters $\lambda^{a}$ are the functions of the target field $A^{+}$defined so that 


$$
\exp \left[i T^{a} \lambda^{a}\left(\mathbf{x}_{i}\right)\right]=W\left(\mathbf{x}_{i}\right)
$$

Various differential cross sections are given by the expectation values of gluonic observables $O\left(a, a^{\dagger}\right)$ in the outgoing wave function at $t \rightarrow \infty$. The evolution between the time of scattering and the time of measurement $(t \rightarrow \infty)$ is unitary, and thus does not affect inclusive observables like total or inelastic cross sections. But it certainly does affect the measured particle spectrum via the final state radiation and has to be taken into account. Therefore expectation values of gluonic observables $O\left(a, a^{\dagger}\right)$ are not simply given by $\left\langle\Psi_{\text {out }}\left|O\left(a, a^{\dagger}\right)\right| \Psi_{\text {out }}\right\rangle$ with $\left|\Psi_{\text {out }}\right\rangle$ of (3.4) since $\left|\Psi_{\text {out }}\right\rangle$ has to be evolved to infinite time before the average can be taken [20]. Another way to view the final state radiation is to realize that the state immediately after the scattering has a nonvanishing overlap with the incoming state $\left|\Psi_{i n}\right\rangle$ and similar "hadronic states". [By hadronic states in this context we mean the states constructed just like the incoming state, so that they are perturbative eigenstates of the QCD Hamiltonian, which in the lowest perturbative order are orthogonal to single gluon states. We do not imply of course that within the present approximation one has to take into account the nonperturbative hadronization process.] This overlap has to be subtracted to get a result which counts only the produced free gluons, and not those which are contained in the incoming wave function or the wave function of outgoing "hadronic" states but not freed during the scattering process.

Consider for example the simplest possible projectile, namely the wave function of a quark at transverse coordinate zero and color index $\alpha$, which is "dressed" to first order in perturbation theory. The incoming quark state contains a component coming from the splitting $\alpha \rightarrow \beta b$, where the gluon labeled by adjoint index $b$ sits at a transverse position displaced by $\mathbf{z}$ from its parent quark. To first order, the quark wave function reads

$$
\begin{aligned}
\left|\alpha_{D}\right\rangle & =|\alpha\rangle+\int d \mathbf{z} d \xi f_{i}(\mathbf{z}) T_{\alpha \beta}^{b}|\beta ; b(\mathbf{z}, i, \xi)\rangle \\
& =|\alpha\rangle+\int d \mathbf{z} \vec{f}(\mathbf{z}) T_{\alpha \beta}^{b}|\beta ; b(\mathbf{z})\rangle .
\end{aligned}
$$

Here, the second line specifies a shorthand used below, and

$$
f_{i}(\mathbf{z})=\frac{g}{2 \pi^{3 / 2}} \frac{z_{i}}{\mathbf{z}^{2}}
$$

denotes the perturbative Weizsäcker-Williams (WW) gluon field and the index $i$ labels the direction in the transverse plane. For the single inclusive gluon cross section, one has to calculate

$$
\left\langle\delta \Psi\left|a_{i}^{d^{\dagger}}(\mathbf{p}) a_{i}^{d}(\mathbf{p})\right| \delta \Psi\right\rangle \quad ; \quad|\delta \Psi\rangle=\left|\Psi_{\text {out }}\right\rangle-\sum_{\alpha}\left|\alpha_{D}\right\rangle\left\langle\alpha_{D} \mid \Psi_{\text {out }}\right\rangle .
$$


The subtraction (3.9) properly accounts for the evolution of the scattered system after it emerges from the target [20], since the only "hadronic state" in the final state to the first order in $\alpha_{s}$ is the dressed quark. Eq.(3.9) ensures that the observable does not include the gluons in the wave function of this dressed quark.

The above example illustrates the general statement that in calculations of $O\left(a, a^{\dagger}\right)$, one should not count gluons that belong to the WW cloud of any of the fast charged partons that constitute the projectile. Rather, a "quark" or a "gluon" in the final state should not be thought of as a free Fock space quark or gluon, but the quark (or gluon) plus its WW cloud. The change of basis from free to dressed partons is described by the unitary "gluon cloud" operator $C[20]$

$$
\left|\alpha_{D}\right\rangle=C|\alpha\rangle
$$

with

$$
C=P \exp \left(i \int d \mathbf{x} d \mathbf{z} d \xi f_{i}(\mathbf{z}-\mathbf{x})\left[a_{i}^{d}(\mathbf{z}, \xi)+a_{i}^{d \dagger}(\mathbf{z}, \xi)\right] \rho_{\xi}^{d}(\mathbf{x})\right)
$$

where $P$ stands for rapidity ordering, and $\rho_{\xi}^{d}(\mathbf{x})$ denotes the total charge density operator integrated from the rapidity of the projectile to $\xi$. The expression (3.11) is only valid to first order in $\alpha_{s}$, but we will not need higher orders in the calculations presented in this paper. The observable

$$
C a_{i}^{d^{\dagger}} a_{i}^{d} C^{\dagger}
$$

counts directly those low $\mathrm{x}$ gluons whose wave functions are orthogonal to those of dressed quarks (and dressed "valence" gluons). Since the dressed gluons are eigenstates of the propagation outside the target, they can be counted directly in $\left|\Psi_{\text {out }}\right\rangle$ without the need to account for the additional unitary evolution to infinite time. This is also true for any other gluonic observable. Thus the correct expression for calculating an arbitrary gluonic observable $O$ in the state $t \rightarrow \infty$ is

$$
\left\langle\Psi_{\text {out }}\left|C O\left(a, a^{\dagger}\right) C^{\dagger}\right| \Psi_{\text {out }}\right\rangle
$$

with the gluon cloud operator $C$ given by Eq.(3.11). One can explicitly verify that this procedure reproduces Eq.(3.9) in the simple example given above. 


\section{Single gluon and quark-gluon inclusive cross sections}

We now apply the formalism of Section 3 to the calculation of the one gluon inclusive emission cross section for an arbitrary projectile which contains a small number of partons, $n \ll 1 / \alpha_{s}$. The restriction to a small number of partons is necessary to treat the projectile wave function perturbatively.

We take the wave function of the projectile in the form of a valence Fock space dressed by the WW field

$$
\left|\Psi_{i n}\right\rangle=C|R\rangle
$$

where $|R\rangle$ is an arbitrary state in the free Fock space with a small number of large rapidity partons. The outgoing wave function after the propagation through the target is

$$
\left|\Psi_{\text {out }}\right\rangle=\hat{W} C|R\rangle \text {. }
$$

The gluon yield per unit rapidity at transverse momentum $\mathbf{p}$ is given by [see Eq. (3.13)]

$$
\frac{d N}{d y d \mathbf{p}}=\left\langle\Psi_{\text {out }}\left|C a_{i}^{d^{\dagger}}(\mathbf{p}, y) a_{i}^{d}(\mathbf{p}, y) C^{\dagger}\right| \Psi_{\text {out }}\right\rangle
$$

To evaluate this, we act with the gluon cloud operator $C$ on the gluon annihilation and creation operators $a$ and $a^{\dagger}$

$$
C a_{i}^{d} C^{\dagger}=a_{i}^{d}-b_{i}^{d}(\rho)
$$

where $b_{i}^{d}(\rho)$ is the classical WW field associated with the charge density $\rho$

$$
b_{i}^{d}(\mathbf{z}, \rho)=\int d \mathbf{x} f_{i}(\mathbf{z}-\mathbf{x}) \rho^{d}(\mathbf{x}) .
$$

To leading order in $\alpha_{s}$, the gluon cloud operator $C$ commutes with the charge density $\rho^{d}$. We thus find

$$
\begin{aligned}
& \left(a_{i}-b_{i}(\rho)\right)\left|\Psi_{\text {out }}\right\rangle=\hat{W} \hat{W}^{\dagger}\left(a_{i}-b_{i}(\rho)\right) \hat{W} C|R\rangle, \\
& \hat{W}\left(w a_{i}-b_{i}(w \rho)\right) C|R\rangle=\hat{W} C\left(w a_{i}-b_{i}(w \rho)\right)|R\rangle .
\end{aligned}
$$

We have used here the fact that the state $|R\rangle$ does not contain gluons with rapidities corresponding to the operator $a$, and hence it is annihilated by $a$. The shorthand notation in (4.7) stands for 


$$
w a_{i}^{d} \equiv W_{d b}^{A}(\mathbf{z}) a_{i}^{b}(\mathbf{z}), \text { etc }
$$

with $W^{A}$ - the eikonal Wilson factor in the adjoint representation. We thus have

$$
\begin{array}{r}
\frac{d N}{d y d \mathbf{p}}=\frac{1}{(2 \pi)^{2}} \int d \mathbf{z} d \overline{\mathbf{z}} e^{-i \mathbf{p} \cdot(\mathbf{z}-\overline{\mathbf{z}})}\langle R|\left[w^{\dagger}(\mathbf{z}) b_{i}^{d}(\mathbf{z}, \rho)-b_{i}^{d}(\mathbf{z}, w \rho)\right] \\
\times\left[w(\overline{\mathbf{z}}) b_{i}^{d}(\overline{\mathbf{z}}, \rho)-b_{i}^{d}(\overline{\mathbf{z}}, w \rho)\right]|R\rangle .
\end{array}
$$

Using

$$
\begin{aligned}
w(\mathbf{z}) b_{i}^{d}(\mathbf{z}, \rho)-b_{i}^{d}(\mathbf{z}, w \rho)= & W_{d b}^{A}(\mathbf{z}) \int d \mathbf{x} f_{i}(\mathbf{z}-\mathbf{x}) \rho^{b}(\mathbf{x}) \\
& -\int d \mathbf{x} f_{i}(\mathbf{z}-\mathbf{x}) W_{d b}^{A}(\mathbf{x}) \rho^{b}(\mathbf{x}),
\end{aligned}
$$

we find

$$
\begin{aligned}
& \frac{d N}{d y d \mathbf{p}}=\frac{\alpha_{s} C_{F}}{\pi^{2}} \frac{1}{(2 \pi)^{2}} \int d \mathbf{z} d \overline{\mathbf{z}} e^{-i \mathbf{p} \cdot(\mathbf{z}-\overline{\mathbf{z}})} \int d \mathbf{x} d \overline{\mathbf{x}}\left\langle\rho^{a}(\mathbf{x}) \rho^{b}(\overline{\mathbf{x}})\right\rangle_{P} \frac{(\mathbf{z}-\mathbf{x}) \cdot(\overline{\mathbf{z}}-\overline{\mathbf{x}})}{(\mathbf{z}-\mathbf{x})^{2}(\overline{\mathbf{z}}-\overline{\mathbf{x}})^{2}} \\
& \left\langle\left[W^{A^{\dagger}}(\mathbf{z}) W^{A}(\overline{\mathbf{z}})+W^{A^{\dagger}}(\mathbf{x}) W^{A}(\overline{\mathbf{x}})-W^{A^{\dagger}}(\mathbf{z}) W^{A}(\overline{\mathbf{x}})-W^{A^{\dagger}}(\mathbf{x}) W^{A}(\overline{\mathbf{z}})\right]^{a b}\right\rangle_{T},
\end{aligned}
$$

where the averages of the charge density and of the products of eikonal factors are taken with respect to the projectile and the target wave functions, respectively. For a translationally invariant and gauge singlet but otherwise arbitrary target this expression reduces to the $k_{T}$-factorized form derived in [32-34].

\section{A. Quark-gluon correlation function: $q A \rightarrow q(\mathbf{k}) g(\mathbf{p}) X$}

We now turn to the simplest two-parton correlation function calculable in the eikonal formalism: a single quark projectile of final transverse momentum $\mathbf{k}$ which shares its recoil between a gluon of transverse momentum $\mathbf{p}$ and the target. The correlation function is

$$
\frac{d N}{d y d \mathbf{k} d \mathbf{p}}=\left\langle\Psi_{\text {out }}\left|C a_{i}^{b^{\dagger}}(\mathbf{p}, y) a_{i}^{b}(\mathbf{p}, y) d_{\delta}^{\dagger}(\mathbf{k}) d_{\delta}(\mathbf{k}) C^{\dagger}\right| \Psi_{\text {out }}\right\rangle_{P}
$$

Here, $d_{\delta}^{\dagger}$ is the quark creation operator at projectile rapidity, and $\left|\Psi_{\text {out }}(\alpha)\right\rangle=$ $\hat{W} C|\alpha\rangle$. We average over the color charge $\alpha$ of the incoming quark,

$$
\left\langle\Psi_{\text {out }}|O| \Psi_{\text {out }}\right\rangle_{P}=\frac{1}{N} \Sigma_{\alpha}\left\langle\Psi_{\text {out }}(\alpha)|O| \Psi_{\text {out }}(\alpha)\right\rangle .
$$


The quark color charge density operator is

$$
\rho^{a}(\mathbf{x})=d_{\alpha}^{\dagger}(\mathbf{x}) T_{\alpha \beta}^{a} d_{\beta}(\mathbf{x}) .
$$

This charge creates the Weizsäcker-Williams field according to Eq.(4.5). The calculation of the previous subsection is easily repeated. The action of the eikonal S-matrix operator $\hat{W}$ amounts to rotating the quark and the gluon creation operators by appropriate eikonal factors. This amounts to attaching a fundamental Wilson line $W_{\beta \delta}^{F}(\mathbf{x})$ at the transverse position of the quark and an adjoint Wilson line $W_{b d}^{A}(\mathbf{z})$ at the transverse position of the gluon. We find

$$
\begin{aligned}
& a_{i}^{d}(\mathbf{z}, y) d_{\delta}(\mathbf{x}) C^{\dagger}\left|\Psi_{\text {out }}(\alpha)\right\rangle \\
& \quad=f_{i}(\mathbf{z}-\mathbf{x})\left[T_{\alpha \beta}^{b} W_{\beta \delta}^{F}(\mathbf{x}) W_{b d}^{A}(\mathbf{z})-T_{\beta \delta}^{d} W_{\alpha \beta}^{F}(\mathbf{x})\right]|\alpha\rangle .
\end{aligned}
$$

In terms of this expression we can write the quark-gluon correlator (4.12) explicitly. In the large- $N$ limit, the color algebra simplifies considerably and one obtains for the two-particle correlation integrated over impact parameter b

$$
\begin{gathered}
\int d \mathbf{b} \frac{d N}{d y d \mathbf{k} d \mathbf{p}}=\frac{1}{(2 \pi)^{4}} \int_{\mathbf{x} \overline{\mathbf{x}} \mathbf{z} \overline{\mathbf{z}}} e^{-i \mathbf{k} \cdot(\mathbf{x}-\overline{\mathbf{x}})-i \mathbf{p} \cdot(\mathbf{z}-\overline{\mathbf{z}})} \vec{f}(\mathbf{z}-\mathbf{x}) \cdot \vec{f}(\overline{\mathbf{z}}-\overline{\mathbf{x}}) \\
\times[Q(\mathbf{z}, \mathbf{x}, \overline{\mathbf{x}}, \overline{\mathbf{z}}) S(\overline{\mathbf{z}}, \mathbf{z})+S(\mathbf{x}, \overline{\mathbf{x}}) \\
-S(\mathbf{x}, \overline{\mathbf{z}}) S(\overline{\mathbf{z}}, \overline{\mathbf{x}})-S(\mathbf{x}, \mathbf{z}) S(\mathbf{z}, \overline{\mathbf{x}})]
\end{gathered}
$$

Here, we use the shorthand $\int_{\mathbf{x}}=\int d \mathbf{x}$. This quark-gluon correlation function is expressed in terms of two target averages, $S(\overline{\mathbf{z}}, \mathbf{z})$ and $Q(\mathbf{z}, \mathbf{x}, \overline{\mathbf{x}}, \overline{\mathbf{z}})$ defined in eqs. (2.2)-(2.6). Integrated over $\mathbf{k}$, it reproduces the expression for the single gluon inclusive emission cross section given in (4.11). This can be checked explicitly by inserting in (4.11) the color charge density correlator $\left\langle\rho^{a}(\mathbf{x}) \rho^{b}(\overline{\mathbf{x}})\right\rangle_{P}=\frac{1}{2 N} \delta^{a b} \delta(\mathbf{x}) \delta(\overline{\mathbf{x}})$ and writing the averages over adjoint Wilson lines in terms of fundamental ones.

\section{The two gluon inclusive cross section: $n A \rightarrow g\left(\mathbf{p}_{1}\right) g\left(\mathbf{p}_{2}\right) X$}

In this section we calculate the eikonal cross section for production of two gluons. We first give the derivation for an arbitrary perturbative projectile. Then we specialize to the case of a single quark projectile.

The cross section for production of two gluons with rapidities and transverse momenta $\left(\eta, \mathbf{p}_{1}\right)$ and $\left(\xi, \mathbf{p}_{2}\right)$ is 


$$
\begin{array}{r}
\frac{d N}{d \eta d \mathbf{p}_{1} d \xi d \mathbf{p}_{2}}=\frac{1}{(2 \pi)^{4}} \int_{\mathbf{z} \overline{\mathbf{z}} \mathbf{u} \mathbf{u}} e^{-i \mathbf{p}_{1} \cdot(\mathbf{z}-\overline{\mathbf{z}})-i \mathbf{p}_{2} \cdot(\mathbf{u}-\overline{\mathbf{u}})}\langle R| C^{\dagger} \hat{W}^{\dagger} C\left[a_{i}^{a \dagger}(\mathbf{z}, \xi) a_{i}^{a}(\overline{\mathbf{z}}, \xi)\right. \\
\left.a_{j}^{b^{\dagger}}(\mathbf{u}, \eta) a_{j}^{b}(\overline{\mathbf{u}}, \eta)\right] C^{\dagger} \hat{W} C|R\rangle
\end{array}
$$

Our convention is such that the rapidity $\eta$ is closer to the rapidities of the valence partons in the projectile, $\eta \gg \xi$. We consider $\eta$ and $\xi$ to be sufficiently close, so that no evolution effects between $\eta$ and $\xi$ have to be taken into account.

For this case, the action of the cloud operator $C$ Eq.(3.11) on the gluon field operator can be restricted to the two rapidities $\eta$ and $\xi$. We can therefore write it schematically as

$$
C=C_{\xi} C_{\eta}
$$

with

$$
\begin{aligned}
& C_{\eta}=\exp \left[i \int_{\mathbf{z}} b_{i}^{d}(\mathbf{z})\left[a_{i}^{d}(\mathbf{z}, \eta)+a_{i}^{d \dagger}(\mathbf{z}, \eta)\right]\right] \\
& C_{\xi}=\exp \left[i \int_{\mathbf{z}}\left[b_{i}^{d}(\mathbf{z})+\delta b_{i}^{d}(\mathbf{z})\right]\left[a_{i}^{d}(\mathbf{z}, \xi)+a_{i}^{d \dagger}(\mathbf{z}, \xi)\right]\right] .
\end{aligned}
$$

Here, $\rho^{d}(\mathbf{x})$ is the charge density operator at the valence rapidity. It determines the classical WW field $b_{i}^{d}(\mathbf{z})$ as specified in Eq. (4.5). The color charges produced at rapidity $\eta$ are measured by $\rho_{\eta}^{d}(\mathbf{x})$ and constitute an additional contribution $\delta b_{i}^{d}(\mathbf{z})$ to the WW field which affects the gluon production at lower rapidities $\xi$,

$$
\delta b_{i}^{d}(\mathbf{z})=\int_{\mathbf{x}} f_{i}(\mathbf{z}-\mathbf{x}) \rho_{\eta}^{d}(\mathbf{x}),
$$

where

$$
\rho_{\eta}^{d}(\mathbf{x})=a_{i}^{b \dagger}(\mathbf{x}, \eta) T_{b c}^{d} a_{i}^{c}(\mathbf{x}, \eta), \quad T_{b c}^{a}=-i f^{a b c} .
$$

To construct a state with up to two gluons, we have to expand the cloud operator (5.2) up to second order in $\rho$,

$$
\begin{gathered}
C=1+i \int_{\mathbf{z}}\left\{\left[a_{i}^{d}(\mathbf{z}, \eta)+a_{i}^{d^{\dagger}}(\mathbf{z}, \eta)\right] b_{i}^{d}(\mathbf{z})\right. \\
\left.+\left[a_{i}^{d}(\mathbf{z}, \xi)+a_{i}^{d^{\dagger}}(\mathbf{z}, \xi)\right]\left[b_{i}^{d}(\mathbf{z})+\delta b_{i}^{d}(\mathbf{z})\right]\right\} \\
-\int_{\mathbf{z}, \mathbf{u}}\left[a_{i}^{d}(\mathbf{z}, \xi)+a_{i}^{d^{\dagger}}(\mathbf{z}, \xi)\right]\left[b_{i}^{d}(\mathbf{z})+\delta b_{i}^{d}(\mathbf{z})\right] \\
\times\left[a_{i}^{d}(\mathbf{u}, \eta)+a_{i}^{d^{\dagger}}(\mathbf{u}, \eta)\right] b_{i}^{d}(\mathbf{u}) .
\end{gathered}
$$


It is now a straightforward albeit tedious matter to calculate $C^{\dagger} \hat{W} C$ and to act with it on $|R\rangle$. [Alternatively, one can construct $\left|\Psi_{\text {in }}\right\rangle$ and $\left|\Psi_{\text {out }}\right\rangle$ from the cloud operator and evaluate the gluon number operator in this $\left|\Psi_{\text {out }}\right\rangle$ state. This is done in Appendix A.] Remembering that $\hat{W}^{\dagger} a_{i}^{a}(\mathbf{z}) \hat{W}=w^{a b}(\mathbf{z}) a_{i}(\mathbf{z})$ and $\hat{W}^{\dagger} \rho^{a}(\mathbf{x}) W=w^{a b}(\mathbf{x}) \rho^{b}(\mathbf{x})$, we get after some algebra

$$
\begin{aligned}
& \hat{W}^{\dagger} a_{i}^{a}(\overline{\mathbf{z}}, \xi) a_{j}^{b}(\overline{\mathbf{u}}, \eta) C^{\dagger} \hat{W} C|R\rangle \\
& =\int_{\overline{\mathbf{x}}_{1}, \overline{\mathbf{x}}_{2}} f_{i}\left(\overline{\mathbf{z}}-\overline{\mathbf{x}}_{1}\right) f_{j}\left(\overline{\mathbf{u}}-\overline{\mathbf{x}}_{2}\right)\left\{\left(w\left(\overline{\mathbf{x}}_{1}\right)-w(\overline{\mathbf{z}})\right) \rho\left(\overline{\mathbf{x}}_{1}\right)\right\}^{a}\left\{w(\overline{\mathbf{u}}) \rho\left(\overline{\mathbf{x}}_{2}\right)\right\}^{b}|R\rangle \\
& -\int_{\overline{\mathbf{x}}_{1}, \overline{\mathbf{x}}_{2}} f_{i}\left(\overline{\mathbf{z}}-\overline{\mathbf{x}}_{1}\right) f_{j}\left(\overline{\mathbf{u}}-\overline{\mathbf{x}}_{2}\right)\left\{w\left(\overline{\mathbf{x}}_{2}\right) \rho\left(\overline{\mathbf{x}}_{2}\right)\right\}^{b}\left\{\left(w\left(\overline{\mathbf{x}}_{1}\right)-w(\overline{\mathbf{z}})\right) \rho\left(\overline{\mathbf{x}}_{1}\right)\right\}^{a}|R\rangle \\
& +\int_{\overline{\mathbf{x}}_{1}} f_{i}(\overline{\mathbf{z}}-\overline{\mathbf{u}}) f_{j}\left(\overline{\mathbf{u}}-\overline{\mathbf{x}}_{1}\right)\left\{(w(\overline{\mathbf{z}})-w(\overline{\mathbf{z}})) w^{\dagger}(\overline{\mathbf{u}}) T^{b} w(\overline{\mathbf{u}}) \rho\left(\overline{\mathbf{x}}_{1}\right)\right\}^{a}|R\rangle
\end{aligned}
$$

With this state, the expectation value of the observable (5.1) can be calculated directly. It contains terms which are quadratic, cubic and quartic in the density of the projectile,

$$
\frac{d N}{d \eta d \mathbf{p}_{1} d \xi d \mathbf{p}_{2}}=\frac{1}{(2 \pi)^{4}} \int_{\mathbf{z} \overline{\mathbf{z}} \mathbf{u}} e^{-i \mathbf{p}_{1} \cdot(\mathbf{z}-\overline{\mathbf{z}})-i \mathbf{p}_{2} \cdot(\mathbf{u}-\overline{\mathbf{u}})}\left[\Sigma_{2}+\Sigma_{3}+\Sigma_{4}\right]
$$

where

$$
\begin{aligned}
\Sigma_{2}= & \int_{\mathbf{x} \overline{\mathbf{x}}} \vec{f}(\mathbf{u}-\mathbf{z}) \cdot \vec{f}(\overline{\mathbf{z}}-\overline{\mathbf{u}}) \vec{f}(\overline{\mathbf{u}}-\overline{\mathbf{x}}) \cdot \vec{f}(\mathbf{x}-\mathbf{u})\left\langle\rho^{a}(\mathbf{x}) \rho^{b}(\overline{\mathbf{x}})\right\rangle_{P} \\
& \times\left\langle\left\{ w^{\dagger}(\mathbf{u}) T^{c} w(\mathbf{u})\left(w^{\dagger}(\mathbf{z})-w^{\dagger}(\mathbf{u})\right)\right.\right. \\
& \left.\left.(w(\overline{\mathbf{z}})-w(\overline{\mathbf{u}})) w^{\dagger}(\overline{\mathbf{u}}) T^{c} w(\overline{\mathbf{u}})\right\}^{a b}\right\rangle \\
\Sigma_{3}= & \int_{\mathbf{x}_{1} \overline{\mathbf{x}}_{1} \overline{\mathbf{x}}_{2}} \vec{f}(\mathbf{u}-\mathbf{z}) \cdot \vec{f}\left(\overline{\mathbf{z}}-\overline{\mathbf{x}}_{1}\right) \vec{f}\left(\mathbf{x}_{1}-\mathbf{u}\right) \cdot \vec{f}\left(\overline{\mathbf{u}}-\overline{\mathbf{x}}_{2}\right) \\
& \times\left[\rho^{c}\left(\mathbf{x}_{1}\right) \rho^{e}\left(\overline{\mathbf{x}}_{2}\right) \rho^{d}\left(\overline{\mathbf{x}}_{1}\right)\right\rangle_{P} \\
& \times\left\langle\left\{\left[w^{\dagger}\left(\overline{\mathbf{x}}_{1}\right)-w^{\dagger}(\overline{\mathbf{z}})\right][w(\mathbf{z})-w(\mathbf{u})] T^{c} w^{\dagger}(\mathbf{u}) w\left(\overline{\mathbf{x}}_{2}\right)\right\}^{d e}\right\rangle_{T} \\
& \quad-\left\langle\rho^{c}\left(\mathbf{x}_{1}\right) \rho^{d}\left(\overline{\mathbf{x}}_{1}\right) \rho^{e}\left(\overline{\mathbf{x}}_{2}\right)\right\rangle_{P} \\
& \left.\times\left\langle\left\{\left[w^{\dagger}\left(\overline{\mathbf{x}}_{1}\right)-w^{\dagger}(\overline{\mathbf{z}})\right][w(\mathbf{z})-w(\mathbf{u})] T^{c} w^{\dagger}(\mathbf{u}) w(\overline{\mathbf{u}})\right\}^{d e}\right\rangle_{T}\right] \\
+ & \int_{\mathbf{x}_{1} \mathbf{x}_{2} \overline{\mathbf{x}}_{1}} \vec{f}\left(\overline{\mathbf{z}}-\overline{\mathbf{u}}^{\prime}\right) \cdot \vec{f}\left(\mathbf{x}_{1}-\mathbf{z}\right) \vec{f}\left(\overline{\mathbf{u}}-\overline{\mathbf{x}}_{1}\right) \cdot \vec{f}\left(\mathbf{x}_{2}-\mathbf{u}\right) \\
& \times\left[\left\langle\rho^{d}\left(\mathbf{x}_{1}\right) \rho^{c}\left(\mathbf{x}_{2}\right) \rho^{e}\left(\overline{\mathbf{x}}_{1}\right)\right\rangle_{P}\right. \\
& \times\left\langle\left\{w^{\dagger}\left(\mathbf{x}_{2}\right) w(\overline{\mathbf{u}}) T^{e}\left[w^{\dagger}(\overline{\mathbf{z}})-w^{\dagger}(\overline{\mathbf{u}})\right]\left[w\left(\mathbf{x}_{1}\right)-w(\mathbf{z})\right]\right\}^{c d}\right\rangle_{T} \\
& \quad\left\langle\rho^{c}\left(\mathbf{x}_{2}\right) \rho^{d}\left(\mathbf{x}_{1}\right) \rho^{e}\left(\overline{\mathbf{x}}_{1}\right)\right\rangle_{P}
\end{aligned}
$$




$$
\left.\times\left\langle\left\{w^{\dagger}(\mathbf{u}) w(\overline{\mathbf{u}}) T^{e}\left[w^{\dagger}(\overline{\mathbf{z}})-w^{\dagger}(\overline{\mathbf{u}})\right]\left[w\left(\mathbf{x}_{1}\right)-w(\mathbf{z})\right]\right\}^{c d}\right\rangle_{T}\right],
$$

and

$$
\begin{aligned}
& \Sigma_{4}=\int_{\mathbf{x}_{1} \mathbf{x}_{2} \overline{\mathbf{x}}_{1} \overline{\mathbf{x}}_{2}} \vec{f}\left(\overline{\mathbf{z}}-\overline{\mathbf{x}}_{1}\right) \cdot \vec{f}\left(\mathbf{x}_{1}-\mathbf{z}\right) \vec{f}\left(\overline{\mathbf{u}}-\overline{\mathbf{x}}_{2}\right) \cdot \vec{f}\left(\mathbf{x}_{2}-\mathbf{u}\right) \\
& \times\left[\left\langle\rho^{g}\left(\mathbf{x}_{2}\right) \rho^{k}\left(\mathbf{x}_{1}\right) \rho^{l}\left(\overline{\mathbf{x}}_{1}\right) \rho^{h}\left(\overline{\mathbf{x}}_{2}\right)\right\rangle_{P}\right. \\
& \quad \times\left\langle\left\{\left[w^{\dagger}\left(\mathbf{x}_{1}\right)-w^{\dagger}(\mathbf{z})\right]\left[w\left(\overline{\mathbf{x}}_{1}\right)-w(\overline{\mathbf{z}})\right]\right\}^{k l}\left\{w^{\dagger}(\mathbf{u}) w(\overline{\mathbf{u}})\right\}^{g h}\right\rangle_{T} \\
&-\left\langle\rho^{k}\left(\mathbf{x}_{1}\right) \rho^{g}\left(\mathbf{x}_{2}\right) \rho^{l}\left(\overline{\mathbf{x}}_{1}\right) \rho^{h}\left(\overline{\mathbf{x}}_{2}\right)\right\rangle_{P} \\
& \times\left\langle\left\{\left[w^{\dagger}\left(\mathbf{x}_{1}\right)-w^{\dagger}(\mathbf{z})\right]\left[w\left(\overline{\mathbf{x}}_{1}\right)-w(\overline{\mathbf{z}})\right]\right\}^{k l}\left\{w^{\dagger}\left(\mathbf{x}_{2}\right) w(\overline{\mathbf{u}})\right\}^{g h}\right\rangle_{T} \\
&-\left\langle\rho^{g}\left(\mathbf{x}_{2}\right) \rho^{k}\left(\mathbf{x}_{1}\right) \rho^{h}\left(\overline{\mathbf{x}}_{2}\right) \rho^{l}\left(\overline{\mathbf{x}}_{1}\right)\right\rangle_{P} \\
& \times\left\langle\left\{\left[w^{\dagger}\left(\mathbf{x}_{1}\right)-w^{\dagger}(\mathbf{z})\right]\left[w\left(\overline{\mathbf{x}}_{1}\right)-w(\overline{\mathbf{z}})\right]\right\}^{k l}\left\{w^{\dagger}(\mathbf{u}) w\left(\overline{\mathbf{x}}_{2}\right)\right\}^{g h}\right\rangle_{T} \\
&+\left\langle\rho^{k}\left(\mathbf{x}_{1}\right) \rho^{g}\left(\mathbf{x}_{2}\right) \rho^{h}\left(\overline{\mathbf{x}}_{2}\right) \rho^{l}\left(\overline{\mathbf{x}}_{1}\right)\right\rangle_{P} \\
&\left.\times\left\langle\left\{\left[w^{\dagger}\left(\mathbf{x}_{1}\right)-w^{\dagger}(\mathbf{z})\right]\left[w\left(\overline{\mathbf{x}}_{1}\right)-w(\overline{\mathbf{z}})\right]\right\}^{k l}\left\{w^{\dagger}\left(\mathbf{x}_{2}\right) w\left(\overline{\mathbf{x}}_{2}\right)\right\}^{g h}\right\rangle_{T}\right] .
\end{aligned}
$$

This is an explicit function of the color charge density correlators in the projectile, and the correlators of the eikonal factors in the target. The term $\Sigma_{2}$ is the probability corresponding to the process when the valence component of $|R\rangle$ emits the gluon with rapidity $\eta$, which subsequently splits into two gluons with rapidities $\eta$ and $\xi$. The term $\Sigma_{4}$ corresponds to the probability of emission of both gluons $\xi$ and $\eta$ from the valence component, and the term $\Sigma_{3}$ is the interference of these two amplitudes. These expressions are general and valid for any perturbative projectile. Also note that we have not used the formal $1 / N_{c}$ expansion to arrive at these expressions. In these aspects, our expression is more general than the one given in [21] for a dipole as projectile.

\section{A. Two-gluon correlations of a single quark projectile: $q A \rightarrow g\left(\mathbf{p}_{1}\right) g\left(\mathbf{p}_{2}\right) X$}

We now specify to the simplest projectile - a single quark. We take the incoming quark to be at the origin of the transverse plane. The color charge density operator is (4.14) and we average again over the color index of the incoming quark as in (4.13). The color charge density correlators are

$$
\begin{aligned}
\left\langle\rho^{a}(\mathbf{x}) \rho^{b}(\mathbf{y})\right\rangle_{P} & =\frac{1}{2 N} \delta^{a b} \delta(\mathbf{x}) \delta(\mathbf{y}) \\
\left\langle\rho^{a}(\mathbf{x}) \rho^{b}(\mathbf{y}) \rho^{c}(\mathbf{z})\right\rangle_{P} & =\frac{1}{4 N}\left(d^{a b c}+i f^{a b c}\right) \delta(\mathbf{x}) \delta(\mathbf{y}) \delta(\mathbf{z}) \\
\left\langle\rho^{a}(\mathbf{x}) \rho^{b}(\mathbf{y}) \rho^{c}(\mathbf{z}) \rho^{d}(\mathbf{u})\right\rangle_{P} & =\left[\frac{1}{2 N^{2}} \delta^{a b} \delta^{c d}+\frac{1}{8 N}\left(d^{a b e}+i f^{a b e}\right)\left(d^{e c d}+i f^{e c d}\right)\right]
\end{aligned}
$$




$$
\times \delta(\mathbf{x}) \delta(\mathbf{y}) \delta(\mathbf{z}) \delta(\mathbf{u})
$$

The two-gluon correlation function (5.1) is given explicitly in terms of these projectile-averaged correlators. In the large- $N_{c}$ limit, the expression simplifies. Using the $S U\left(N_{c}\right)$-identities compiled in the appendix of [20], the two-gluon correlation function can be expressed in terms of the target averages (2.2) and (2.3) of two and four fundamental Wilson lines, respectively. The final result takes the form

$$
\begin{aligned}
& \frac{d N}{d \eta d \mathbf{p}_{1} d \xi d \mathbf{p}_{2}}=\frac{1}{(2 \pi)^{4}} \int_{\mathbf{z} \overline{\mathbf{z}} \mathbf{u} \overline{\mathbf{u}}} e^{-i \mathbf{p}_{1} \cdot(\mathbf{z}-\overline{\mathbf{z}})-i \mathbf{p}_{2} \cdot(\mathbf{u}-\overline{\mathbf{u}})} \vec{f}(\mathbf{u}) \cdot \vec{f}(\overline{\mathbf{u}}) \\
& \times\left[2 \vec { f } ( \mathbf { z } - \mathbf { u } ) \cdot \vec { f } ( \overline { \mathbf { z } } - \overline { \mathbf { u } } ) \left\{Q(\overline{\mathbf{u}}, \mathbf{u}, \mathbf{z}, \overline{\mathbf{z}}) S(\mathbf{z}, \overline{\mathbf{z}}) S(\mathbf{u}, \overline{\mathbf{u}})+S^{2}(\mathbf{u}, \overline{\mathbf{u}})\right.\right. \\
& -S(\overline{\mathbf{u}}, \overline{\mathbf{z}}) S(\overline{\mathbf{z}}, \mathbf{u}) S(\mathbf{u}, \overline{\mathbf{u}})-S(\mathbf{u}, \mathbf{z}) S(\mathbf{z}, \overline{\mathbf{u}}) S(\overline{\mathbf{u}}, \mathbf{u})\} \\
& -\vec{f}(\mathbf{z}-\mathbf{u}) \cdot \vec{f}(\overline{\mathbf{z}})\{Q(\mathbf{u}, \overline{\mathbf{u}}, \overline{\mathbf{z}}, \mathbf{z}) S(\mathbf{u}, \overline{\mathbf{u}}) S(\mathbf{z}, \overline{\mathbf{z}})+S(\mathbf{u}) S(\overline{\mathbf{u}}) S(\mathbf{u}, \overline{\mathbf{u}}) \\
& -Q(\mathbf{u}, \overline{\mathbf{u}}, \mathbf{0}, \mathbf{z}) S(\mathbf{z}) S(\mathbf{u}, \overline{\mathbf{u}})-S(\overline{\mathbf{u}}, \mathbf{u}) S(\mathbf{u}, \overline{\mathbf{z}}) S(\overline{\mathbf{z}}, \overline{\mathbf{u}}) \\
& +Q(\mathbf{u}, \mathbf{0}, \overline{\mathbf{z}}, \mathbf{z}) S(\mathbf{z}, \overline{\mathbf{z}}) S(\mathbf{u})+S^{2}(\mathbf{u}) \\
& -S(\mathbf{u}) S(\mathbf{z}) S(\mathbf{z}, \mathbf{u})-S(\mathbf{u}) S(\overline{\mathbf{z}}, \mathbf{u}) S(\overline{\mathbf{z}})\} \\
& -\vec{f}(\mathbf{z}) \cdot \vec{f}(\overline{\mathbf{z}}-\overline{\mathbf{u}})\{Q(\overline{\mathbf{u}}, \mathbf{u}, \mathbf{z}, \overline{\mathbf{z}}) S(\mathbf{u}, \overline{\mathbf{u}}) S(\mathbf{z}, \overline{\mathbf{z}})+S(\mathbf{u}) S(\overline{\mathbf{u}}) S(\mathbf{u}, \overline{\mathbf{u}}) \\
& -Q(\overline{\mathbf{u}}, \mathbf{u}, \mathbf{0}, \overline{\mathbf{z}}) S(\overline{\mathbf{z}}) S(\mathbf{u}, \overline{\mathbf{u}})-S(\overline{\mathbf{u}}, \mathbf{u}) S(\mathbf{z}, \overline{\mathbf{u}}) S(\mathbf{z}, \mathbf{u}) \\
& +Q(\overline{\mathbf{u}}, \mathbf{0}, \mathbf{z}, \overline{\mathbf{z}}) S(\mathbf{z}, \overline{\mathbf{z}}) S(\overline{\mathbf{u}})+S^{2}(\overline{\mathbf{u}}) \\
& -S(\overline{\mathbf{u}}) S(\overline{\mathbf{z}}) S(\overline{\mathbf{z}}, \overline{\mathbf{u}})-S(\overline{\mathbf{u}}) S(\mathbf{z}) S(\mathbf{z}, \overline{\mathbf{u}})\} \\
& +\vec{f}(\mathbf{z}) \cdot \vec{f}(\overline{\mathbf{z}})\left\{Q(\overline{\mathbf{u}}, \mathbf{u}, \mathbf{z}, \overline{\mathbf{z}}) S(\mathbf{z}, \overline{\mathbf{z}}) S(\mathbf{u}, \overline{\mathbf{u}})+S^{2}(\mathbf{u}, \overline{\mathbf{u}})\right. \\
& -Q(\overline{\mathbf{u}}, \mathbf{u}, \mathbf{0}, \overline{\mathbf{z}}) S(\mathbf{u}, \overline{\mathbf{u}}) S(\overline{\mathbf{z}})+S^{2}(\mathbf{z}, \overline{\mathbf{z}}) \\
& \left.\left.-Q(\overline{\mathbf{u}}, \mathbf{u}, \mathbf{z}, \mathbf{0}) S(\mathbf{u}, \overline{\mathbf{u}}) S(\mathbf{z})+1-S^{2}(\overline{\mathbf{z}})-S^{2}(\mathbf{z})\right\}\right] \text {. }
\end{aligned}
$$

We note that the two gluon correlation function of the perturbative $q \bar{q}$ dipole projectile was derived in Ref. [21] in the large $N_{c}$ limit. We have checked that in the limit of arbitrary large dipole size, the final result of Ref. [21] coincides with (5.14).

\section{Conclusions}

In this paper, we have shown that the framework of perturbative saturation provides for a mechanism which can shift the maximum strength which of azimuthal particle correlations away from $180^{\circ}$. We understand the physics of this phenomenon in the following way. There are two basic mechanisms by which a quark and a gluon can be produced in the final state. The first one 
is the QCD analog of Molière scattering. The quark in the projectile wave function scatters from the perturbative part of the target gluon field and then radiates a gluon in the final state. This final state radiation is predominantly back-to-back and generates maximal correlations at angle $\pi$. The second mechanism is the multiple scattering of the $q g$ component of the initial state on the target. The final states produced by this mechanism depend strongly on the separation between the initial quark and gluon in the transverse plane. For components with large separation (small relative transverse momentum $|\mathbf{k}-\mathbf{p}| \ll Q_{s}$ in the initial state), the quark and the gluon scatter independently off the target fields. This process produces uncorrelated $q g$ pairs in the final state and leads to the broadening of the angular distribution. However, the components of the incoming state which have transverse size smaller than the correlation length of the target fields scatter coherently, i.e. they scatter effectively as a single particle. These quark-gluon components pick up a typical soft momentum (of order $Q_{s, 0}$, as explained in Section 3), which is equally shared between the two partons. As a result, the two partons emerge from the interaction region with momenta shifted in the same direction by an equal amount of order $Q_{s, 0} / 2$. In the initial state, the total transverse momentum vanishes, and thus the momenta of the quark and the gluon are balanced. The equal momentum transfer therefore produces angular correlation in the final state which is peaked away from $180^{\circ}$. The maximum correlation produced by this mechanism for large trigger momenta $k$ can be simply estimated to lie about $Q_{s, 0} / \sqrt{2} k$ away from $180^{\circ}$. Whether this shift in the maximal correlation angle is actually observable in the spectrum depends very much on the relative importance of the Molière component and the coherent soft scattering component. Our numerical results suggest that the coherent scattering component is dominant for the trigger momenta around $Q_{s}$.

We also observe that the coherent scattering component, which shifts the maximal correlation strength away from $180^{\circ}$, is enhanced and dominates over a wider range of trigger momenta, if the projectile system has smaller transverse size. To establish this statement, we use the fact that our calculation allows us to regulate the transverse size of the incoming projectile via the cutoff $\Lambda_{\text {cut }}$. Although it is difficult to draw firm phenomenological conclusions, we believe that there is potential for an observable effect.

Also, it would be interesting to study how this picture discussed above is affected by low- $x$ evolution. One may expect that since $Q_{s}$ increases with rapidity, the maximal correlation angle at forward rapidities should move further away from $180^{\circ}$. However, low- $x$ evolution does not only affect the value of the saturation scale, but also changes significantly the efficiency of the target as a function of momentum transfer. In particular, the momentum dependence of the target gluon distribution decreases slower at large momenta thus effectively increasing the Molière hard scattering component. Hence, it is not clear to us how the azimuthal shift of the maximal two-parton correlation strength 
will evolve with $x$.

\section{A. Alternative calculation of two-gluon correlation function (5.14)}

In this appendix, we derive the two-gluon correlation function (5.14) following the formulation given in $[13,20]$. We start from the state $\left|\psi_{\text {in }}^{\alpha}\right\rangle$ in $(3.1)$ of a single quark with color $\alpha$ and its gluon cloud, expanded in perturbation theory up to $O\left(g^{2}\right)$,

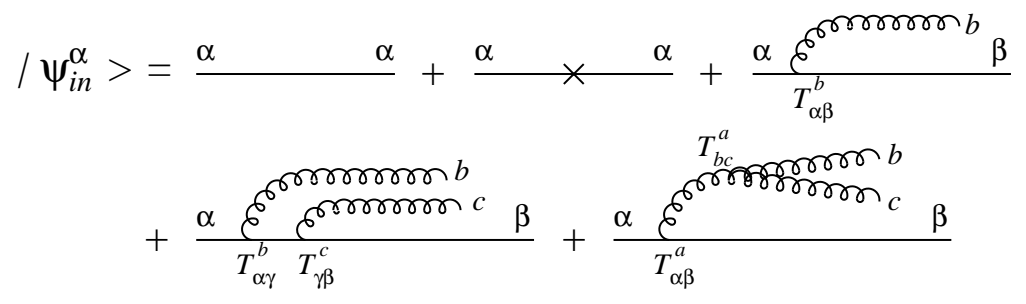

This is the incoming state with two gluons of color $b$ and $c$. The crossed line indicates a probability conserving virtual correction, such that the state $\left|\psi_{\text {in }}^{\alpha}\right\rangle$ is normalized to unity up to $O\left(g^{2}\right)$. In this approximation, the states do not depend on rapidity, and the rapidity labels are suppressed in the following.

The interaction of $\left|\psi_{\text {in }}^{\alpha}\right\rangle$ with the target results in phase shifts by eikonal Wilson lines, which are in the fundamental $\left(W^{F}\right)$ and adjoint $\left(W^{A}\right)$ representation, respectively. This leads to the outgoing state

$$
\begin{aligned}
\left|\Psi_{\text {out }}^{\alpha}\right\rangle= & \left(1-\frac{C_{F}}{2} \int d \mathbf{x} \vec{f}(\mathbf{x}) \cdot \vec{f}(\mathbf{x})\right) W_{\alpha \beta}^{F}(\mathbf{0})|\beta\rangle \\
& +i \int d \mathbf{x} \vec{f}(\mathbf{x})\left(T^{a} W^{F}(\mathbf{0})\right)_{\alpha \beta} W_{a b}^{A}(\mathbf{x})|\beta ; b(\mathbf{x})\rangle \\
& -\frac{1}{2} \int d \mathbf{x} d \mathbf{y}\left[\vec{f}(\mathbf{x}) \vec{f}(\mathbf{y})\left(T^{a} T^{d} W^{F}(\mathbf{0})\right)_{\alpha \beta} W_{a b}^{A}(\mathbf{x}) W_{d c}^{A}(\mathbf{y})\right. \\
& \left.+\vec{f}(\mathbf{x}) \vec{f}(\mathbf{y}-\mathbf{x})\left(T^{e} W^{F}(\mathbf{0})\right)_{\alpha \beta}\left(T_{a d}^{e} W_{a b}^{A}(\mathbf{x}) W_{d c}^{A}(\mathbf{y})\right)\right]|\beta ; b(\mathbf{x}), c(\mathbf{y})\rangle .
\end{aligned}
$$

The quark propagates at the transverse position $\mathbf{x}_{q}=0$, and the gluons propagate at $\mathbf{x}$ and $\mathbf{y}$, respectively.

According to (3.9), we have to subtract the overlap with the dressed incoming state. This leads to the state $\left|\delta \Psi_{\alpha}\right\rangle$ which to lowest order in $\vec{f} \vec{f}$ consists of the following contributions: 


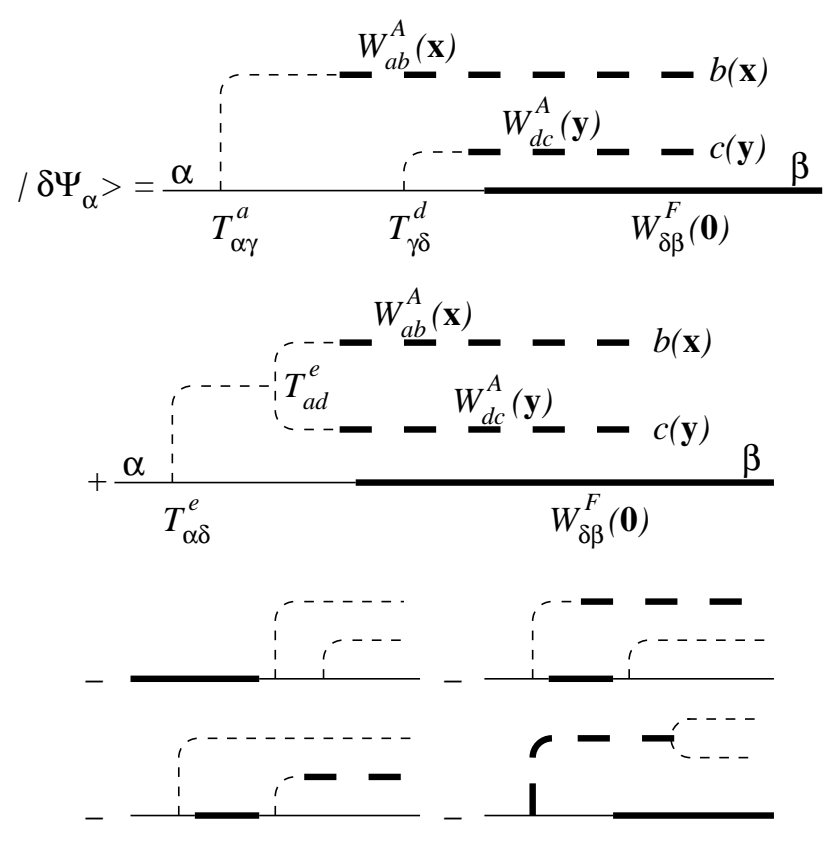

Here, we use the diagrammatic shorthand of Ref. [20] in which thick lines denote partons propagating through the target and thus picking up eikonal Wilson lines in the corresponding representation. The first two graphs correspond to the case that the quark emits both gluons before the target and all three partons propagate through the target (thick lines). The third diagram can be viewed as emission after interaction since the outgoing lines do not carry eikonal factors (thin lines). This third diagram, as well as the remaining three arise from the subtraction of the overlap in (3.9).

A convenient expression for $\left|\delta \Psi_{\alpha}\right\rangle$ is obtained by applying identities like

$$
\left(T^{b} W^{F}(\mathbf{0}) T^{d}\right)_{\alpha \beta}=\left(T^{b} T^{a} W^{F}(\mathbf{0})\right)_{\alpha \beta} W_{a d}^{A}(\mathbf{0}) .
$$

This allows us to shift the Wilson operator $W^{F}(\mathbf{0})$ such that we can write

$$
\begin{aligned}
\left|\delta \Psi_{\alpha}\right\rangle=- & d \mathbf{x} d \mathbf{y} \vec{f}(\mathbf{x}) \vec{f}(\mathbf{y})\left(T^{a} T^{d} W^{F}(\mathbf{0})\right)_{\alpha \beta}\left[W_{a b}^{A}(\mathbf{x})\left(W_{d c}^{A}(\mathbf{0})-W_{d c}^{A}(\mathbf{y})\right)\right. \\
& \left.-W_{d b}^{A}(\mathbf{0})\left(W_{a c}^{A}(\mathbf{0})-W_{a c}^{A}(\mathbf{y})\right)\right]|\beta ; b(\mathbf{x}), c(\mathbf{y})\rangle \\
+ & d \mathbf{x} d \mathbf{y} \vec{f}(\mathbf{x}) \vec{f}(\mathbf{y}-\mathbf{x})\left(T^{e} W^{F}(\mathbf{0})\right)_{\alpha \beta}\left[T_{a d}^{e} W_{a b}^{A}(\mathbf{x}) W_{d c}^{A}(\mathbf{y})\right. \\
& \left.\quad-W_{e a}^{A}(\mathbf{x}) T_{b c}^{a}\right]|\beta ; b(\mathbf{x}), c(\mathbf{y})\rangle .
\end{aligned}
$$

This expression may be written in the shorthand form, introducing an operator $\mathcal{O}(\mathbf{x}, \mathbf{y})$ as follows 


$$
\left|\delta \Psi_{\alpha}\right\rangle=\int d \mathbf{x} d \mathbf{y} \mathcal{O}_{\alpha \beta}^{b c}(\mathbf{x}, \mathbf{y})|\beta ; b(\mathbf{x}), c(\mathbf{y})\rangle
$$

This allows us to write the two-gluon correlation in a compact way by calculating the expectation value of the two-particle number operator in this state, averaged over the incoming quark color index $\alpha$,

$$
\begin{aligned}
& \frac{1}{N} \sum_{\alpha}\left\langle\delta \Psi_{\alpha}\left|a^{\dagger}\left(\mathbf{p}_{1}, \xi\right) a\left(\mathbf{p}_{1}, \xi\right) a^{\dagger}\left(\mathbf{p}_{2}, \eta\right) a\left(\mathbf{p}_{2}, \eta\right)\right| \delta \Psi_{\alpha}\right\rangle \\
& =\frac{1}{(2 \pi)^{4}} \int_{\mathbf{z} \overline{\mathbf{z}} \mathbf{u} \bar{u}} e^{-i \mathbf{p}_{1} \cdot(\mathbf{z}-\overline{\mathbf{z}})-i \mathbf{p}_{2} \cdot(\mathbf{u}-\overline{\mathbf{u}})}\left\langle\left[\mathcal{O}_{\alpha \beta}^{b c}(\overline{\mathbf{z}}, \overline{\mathbf{u}})\right]^{\dagger} \mathcal{O}_{\alpha \beta}^{b c}(\mathbf{z}, \mathbf{u})\right\rangle_{T}
\end{aligned}
$$

Working out the target averages as described in [20], we obtain after some color algebra the result given in (5.14).

1. I. Arsene et al. [BRAHMS Collaboration], arXiv:nucl-ex/0410020.

2. B. B. Back et al. [PHOBOS Collaboration], arXiv:nucl-ex/0410022.

3. K. Adcox et al. [PHENIX Collaboration], arXiv:nucl-ex/0410003.

4. J. Adams et al. [STAR Collaboration], arXiv:nucl-ex/0501009.

5. A. H. Mueller, Lectures given at NATO Advanced Study Institute on Particle Production Spanning MeV and TeV Energies (Nijmegen 99), Nijmegen, Netherlands, 8-20 Aug 1999, arXiv:hep-ph/9911289.

6. J. P. Blaizot and F. Gelis, Nucl. Phys. A 750 (2005) 148.

7. L. McLerran, Nucl. Phys. A 752, 355 (2005).

8. E. Iancu and R. Venugopalan, in "Quark Gluon Plasma 3", (editors: R.C. Hwa and X.N. Wang, World Scientific, Singapore), p.249-363, arXiv:hep$\mathrm{ph} / 0303204$.

9. H. Weigert, arXiv:hep-ph/0501087.

10. J. Jalilian-Marian and Y. V. Kovchegov, arXiv:hep-ph/0505052.

11. P. Jacobs and X. N. Wang, Prog. Part. Nucl. Phys. 54, 443 (2005).

12. R. Baier, D. Schiff and B. G. Zakharov, Ann. Rev. Nucl. Part. Sci. 50, 37 (2000).

13. A. Kovner and U. A. Wiedemann, in "Quark Gluon Plasma 3", (editors: R.C. Hwa and X.N. Wang, World Scientific, Singapore), P.192-248, arXiv:hep$\mathrm{ph} / 0304151$.

14. M. Gyulassy, I. Vitev, X. N. Wang and B. W. Zhang, in "Quark Gluon Plasma 3", (editors: R.C. Hwa and X.N. Wang, World Scientific, Singapore), p. 123191, arXiv:nucl-th/0302077.

15. C. A. Salgado and U. A. Wiedemann, Phys. Rev. Lett. 93, 042301 (2004).

16. N. Armesto, C. A. Salgado and U. A. Wiedemann, Phys. Rev. Lett. 93, 242301 (2004).

17. J. Casalderrey-Solana, E. V. Shuryak and D. Teaney, arXiv:hep-ph/0411315. 
18. E. V. Shuryak, Nucl. Phys. A 750 (2005) 64.

19. D. Kharzeev, E. Levin and L. McLerran, Nucl. Phys. A 748 (2005) 627.

20. A. Kovner and U. A. Wiedemann, Phys. Rev. D 64 (2001) 114002.

21. J. Jalilian-Marian and Y. V. Kovchegov, Phys. Rev. D 70, 114017 (2004) [Erratum-ibid. D 71, 079901 (2005)].

22. N. N. Nikolaev and W. Schäfer, Phys. Rev. D 71 (2005) 014023.

23. N. N. Nikolaev, W. Schäfer, B. G. Zakharov and V. R. Zoller, arXiv:hep$\mathrm{ph} / 0504057$.

24. H. Fujii, F. Gelis and R. Venugopalan, arXiv:hep-ph/0504047.

25. R. Baier, A. Kovner and U. A. Wiedemann, Phys. Rev. D 68, 054009 (2003).

26. D. Kharzeev, E. Levin and L. McLerran, Phys. Lett. B 561, 93 (2003).

27. J. L. Albacete, N. Armesto, A. Kovner, C. A. Salgado and U. A. Wiedemann, Phys. Rev. Lett. 92, 082001 (2004).

28. J. Jalilian-Marian, Y. Nara and R. Venugopalan, Phys. Lett. B 577, 54 (2003).

29. D. Kharzeev, Y. V. Kovchegov and K. Tuchin, Phys. Rev. D 68, 094013 (2003).

30. Y. V. Kovchegov and A. H. Mueller, Nucl. Phys. B 529 (1998) 451.

31. H. A. Bethe, Phys. Rev. D89 (1953) 1256.

32. Y. V. Kovchegov and K. Tuchin, Phys. Rev. D 65, 074026 (2002).

33. A. Dumitru and L. D. McLerran, Nucl. Phys. A 700, 492 (2002).

34. J. P. Blaizot, F. Gelis and R. Venugopalan, Nucl. Phys. A 743, 13 (2004). 